\title{
Implementation and comparison of the recent three-dimensional radiation stress theory and vortex-force formalism in an unstructured-grid coastal circulation model
}

\author{
Meng Xia $^{\mathrm{a}, *}$, Miaohua Mao ${ }^{\mathrm{b}}$, Qianru Niu ${ }^{\mathrm{b}, \mathrm{c}}$

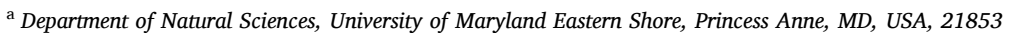 \\ ${ }^{\mathrm{b}}$ Yantai Institute of Coastal Zone Research, Chinese Academy of Sciences, Yantai, 26400, China \\ ${ }^{\mathrm{c}}$ College of Life Sciences and Oceanography, Shenzhen University, Shenzhen, 518060, China
}

\section{A R T I C L E I N F O}

\section{Keywords:}

Wave-induced circulation

Wave-current interaction

Numerical modeling

Radiation stress

Vortex-force formalism

\begin{abstract}
A B S T R A C T
Given the importance of wave-current interaction in estuarine and coastal dynamics, it is crucial to revisit impacts of surface gravity waves on three-dimensional (3D) nearshore circulation. This work investigates waveinduced circulation in three typical coastal systems including an idealized inlet and planar and natural barred beaches, by implementing the recent 3D radiation stress (RS) theory and vortex-force (VF) formalism to an unstructured-grid Finite-Volume Community Ocean Model (FVCOM). In the idealized inlet case, 3D RS generated appreciable currents near barriers and lateral boundaries while VF forced strong flows via breaking and rollerinduced accelerations in front of the inlet. Both simulations indicate vertically varying wave-induced circulation that decreases markedly. In the planar beach with obliquely incident waves, both methods successfully produced surface onshore and bottom undertow, as well as the wave breaking and roller-induced longshore currents. Nevertheless, 3D RS generated unrealistic offshore currents close to the shoreline. The coupled models were validated against observations in the natural barred beach, and results indicate that the 3D RS model agrees slightly better with the observed longshore currents while 3D VF captures the vertical shear of the onshore-offshore flows reasonably. Further investigations suggest that both methods produce the wave breakinginduced surface onshore and bottom undertow successfully, yet they are located further offshore resulting from the 3D RS-induced unrealistic offshore currents. Successful implementations of the paired wave-current theories to the unstructured model would be fundamental and beneficial to the coastal ocean modeling community.
\end{abstract}

\section{Introduction}

Wind generates waves and currents concurrently, and the consequent interaction between the rapidly oscillating surface gravity waves and slowly varying oceanic currents become a key process in coastal dynamics (Benetazzo et al., 2013; Dodet et al., 2013; Mao and Xia, 2017; Niu and Xia, 2017). Specifically, waves modulate air-sea momentum transfers by changing the sea surface roughness (Donelan et al., 1993), contribute to the bottom frictional stress via wave-induced orbital velocities (Madsen, 1994), and generate excessive momentum fluxes (Longuet-Higgins and Stewart, 1964; Longuet-Higgins, 1970).

Given the significance of wave-induced momentum fluxes, corresponding theories have been developed and discussed intensively over the past half century. Initially, Longuet-Higgins and Stewart (1962, 1964) proposed that excessive momentum fluxes resulting from the phase-averaged wave radiation stress (RS) play an important role in the wave-induced dynamics. Alternatively, phase-resolving two-dimensional (2D) Boussinesq (Chen et al., 1999; Kennedy et al., 2002) and Reynolds-Averaged Navier-Stokes Volume of Fluid (RANS-VOF) models (Lin and Liu, 1998; Michaud et al., 2012) were developed to depict wave-current interaction in a more comprehensive but sophisticated way (e.g., resolving the nearshore wave evolution processes). Given that the phase-resolving model is computationally expensive, its applications were mostly limited to short-term, small-scale or idealized current evolution processes. Therefore, 2D RS (Longuet-Higgins and Stewart, 1962, 1964; Phillips, 1977) based models have been successfully used to

\footnotetext{
* Corresponding author.

E-mail address: mxia@umes.edu (M. Xia).
} 
simulate wave-induced circulation in various realistic scenarios (Nielsen and Apelt, 2003; Brown et al., 2013).

Given the complexity of 3D nearshore and coastal circulation (e.g., wave-induced longshore current), Mellor (2003) derived the vertically dependent 3D RS approach, which has been corrected and improved during the last two decades (Mellor, 2003, 2005; 2008, 2013; 2015). Meanwhile, it has been applied to investigate wave-induced dynamics in shallow and coastal regions, including the idealized inlet ( $\mathrm{Wu}$ et al., 2011), natural lagoon (Mao and Xia, 2018) and lakes (Mao and Xia, 2017; Niu and Xia, 2017). It has been proved that 3D RS based models produced comparably or better nearshore hydrodynamics than those forced by 2D RS (Bolaños et al., 2014). Nevertheless, the 3D RS approach of Mellor $(2003$, 2005) was criticized for the correctness of its expression in representing the vertical wave momentum flux (Ardhuin et al., 2008) and the practicability over the sloping bottom (Aiki and Greatbatch, 2013). The abovementioned issue was addressed by Mellor (2013, 2015), which corrected the improper treatment of the pressure term and rectified the unrealistic, surface-intensified force in the vertical flux term. Ardhuin et al. (2017) emphasized that the Airy wave theory based, phase-averaged equations presumed in deriving the 3D RS expression are strictly valid only in systems with a flat bottom, while Mellor (2017) argued that this treatment is appropriate as it restricts the errors to an order of $10^{-2}$ or less and can be used in most oceanographic applications. Alternatively, the vortex-force (VF) formalism based model, which adopted the asymptotic theory (Craik and Leibovich, 1976; McWilliams et al., 2004), gained the popularity by its successful applications to the surf zone and inner shelf (Uchiyama et al., 2010; Kumar et al., 2012). However, a comparison made by Mellor (2016) indicates that the 3D VF is negligibly weaker than the 3D RS. When the momentum balance integrated vertically from the 3D RS approach, its expression coincides mathematically with the generally accepted 2D RS theory (Longuet-Higgins and Stewart, 1962, 1964; Phillips, 1977).

Despite the scientific debate over the correctness and practicability between the 3D RS and VF methods, both concepts were adopted by coastal and ocean communities in modeling wave-induced circulation (Wang and Shen, 2011; Kumar et al., 2012). However, a direct comparison between the paired theories applied to numerical models has been barely made. Uchiyama et al. (2010) found that the two methods produced moderately different currents in a planar beach case, whereas the depth-integrated wave-averaged results (e.g., Eulerian undertow currents) were reported to be similar in flume experiments by Moghimi et al. (2013). Although previous findings are encouraging, additional investigations are required to further validate performances of both methods in producing longshore and cross-shore currents. Moreover, the 3D RS (Mellor, 2003) adopted by Uchiyama et al. (2010) was reported to generate erroneous offshore currents (Kumar et al., 2011). Furthermore, previous applications were limited to structured-grid models (Uchiyama et al., 2010; Kumar et al., 2012; Moghimi et al., 2013), which may not capture the complex nearshore and coastal dynamics sufficiently. Additionally, successful implementations of the unstructured-grid model are fundamental and important for further applications to the complicated and realistic coastal seas with an exceptional computational cost.

In this study, the recently corrected 3D RS formulation (Mellor, 2015) and VF theory (McWilliams et al., 2004) were implemented in an unstructured-grid Finite-Volume Community Ocean Model (FVCOM). Wave-induced currents were compared between the paired theories in three typical coastal cases, including an idealized inlet (Wu et al., 2011), planar beach (Haas and Warner, 2009), and natural barred beach (Newberger and Allen, 2007). Nearshore physics (e.g., wave-induced longshore currents, surface onshore and bottom undertow in the surf zone) revealing the variability between the paired methods were examined and discussed. Remaining sections of this paper are organized as follows: Section 2 introduces the methodology applied in the modeling system; Section 3 describes results and gives discussion; Section 4 summarizes the key findings of this paper with conclusions.

\section{Methodology}

\subsection{Descriptions of the modeling system}

The FVCOM is a free surface, 3D, primitive equation model originally developed by Chen et al. (2013), which has been extensively applied to coastal oceans, estuaries, lagoons and large lakes (Niu et al., 2015; Jiang and Xia, 2016; Kang et al., 2017; Chen et al., 2019; Liu et al., 2019). It adopts unstructured triangular meshes and sigma coordinates at the horizontal and vertical space, respectively, so that the complicated coastlines and highly variable nearshore bathymetry were well captured. The modified Mellor and Yamada level 2.5 scheme (Galperin et al., 1988) and Smagorinsky (1963) turbulent closure parameterizations are adopted to calculate the vertical and horizontal mixing processes. Hydrostatic and Boussinesq assumptions are utilized, in which density variations are neglected, except for the term multiplied by gravity in the buoyancy force.

The Surface Wave Model (SWAVE) is a third-generation wave model (Qi et al., 2009), which has been widely used to resolve wave dynamics in the coastal oceans, Great Lakes, and Arctic Ocean (Niu and Xia, 2016; Mao and Xia, 2017; Zhang et al., 2020). It defines wave evolution processes in deep and shallow waters by solving the wave action balance. This governing equation includes the wind-induced wave generation, wave propagation, three and four-wave nonlinear interaction, white-capping, bottom frictional dissipation, and depth-induced breaking. The frequency and directional space are discretized with the flux-corrected transport algorithm and an implicit Crank-Nicolson solver, respectively. An implicit second-order upwind finite-volume scheme is employed in the geographic space. Detailed model descriptions are referred to Qi et al. (2009) and Mao and Xia (2017).

Although the focus of this work is on the wave-induced processes, impacts of current and depth variations on wave dynamics (e.g., currentinduced refraction and Doppler frequency shift, and depth-induced wave breaking) were included in the wave-current coupled modeling system to maintain the integrality of the two-way dynamic processes. Detailed descriptions of the wave-induced momentum fluxes using the RS and VF approaches in the modeling system were introduced in the following subsections.

\subsection{Wave-induced momentum fluxes}

\subsubsection{Radiation stress formulations}

In the generalized terrain-following sigma coordinates, governing equations of the continuity and momentum balance with the inclusion of radiation stress (RS) terms are given as follows:

$$
\begin{aligned}
& \frac{\partial D U}{\partial x}+\frac{\partial D V}{\partial y}+\frac{\partial \Omega}{\partial \widehat{\sigma}}+\frac{\partial \eta}{\partial t}=0 \\
& \frac{\partial U D}{\partial t}+\frac{\partial U^{2} D}{\partial x}+\frac{\partial U V D}{\partial y}+\frac{\partial U \Omega}{\partial \widehat{\sigma}}-f V D \\
& =-D \frac{\partial}{\partial x}\left(g \eta+p_{a t m}\right)-D \int_{\widehat{\sigma}}^{0}\left(D \frac{\partial \rho}{\partial x}-\widehat{\sigma} \frac{\partial D}{\partial x} \frac{\partial \rho}{\partial \widehat{\sigma}}\right) d \widehat{\sigma} \\
& -\left(\frac{\partial D S_{x x}}{\partial x}+\frac{\partial D S_{x y}}{\partial y}\right)+\widehat{\sigma}\left(\frac{\partial D}{\partial x} \frac{\partial S_{x x}}{\partial \widehat{\sigma}}+\frac{\partial D}{\partial y} \frac{\partial S_{x y}}{\partial \widehat{\sigma}}\right)+\frac{\partial \tau_{x}}{\partial \widehat{\sigma}} \\
& \frac{\partial V D}{\partial t}+\frac{\partial U V D}{\partial x}+\frac{\partial V^{2} D}{\partial y}+\frac{\partial V \Omega}{\partial \widehat{\sigma}}+f U D \\
& =-D \frac{\partial}{\partial y}\left(g \eta+p_{a t m}\right)-D \int_{\widehat{\sigma}}^{0}\left(D \frac{\partial \rho}{\partial y}-\widehat{\sigma} \frac{\partial D}{\partial y} \frac{\partial \rho}{\partial \widehat{\sigma}}\right) d \widehat{\sigma} \\
& -\left(\frac{\partial D S_{x y}}{\partial x}+\frac{\partial D S_{y y}}{\partial y}\right)+\widehat{\sigma}\left(\frac{\partial D}{\partial x} \frac{\partial S_{x y}}{\partial \widehat{\sigma}}+\frac{\partial D}{\partial y} \frac{\partial S_{y y}}{\partial \widehat{\sigma}}\right)+\frac{\partial \tau_{y}}{\partial \widehat{\sigma}}
\end{aligned}
$$

where $x, y, \widehat{\sigma}$ are the east-west, north-south, and vertical axes; $t$ is the time; $(U, V, \Omega)$ are the Lagrangian velocities that combine Eulerian components with Stokes drifts in the $(x, y, \widehat{\sigma})$ space; $\left(\tau_{x}, \tau_{y}\right)$ are the $(x, y)$ 
components of the stress. The total water depth is $D=h+\hat{\eta}$, where $h$ and $\hat{\eta}$ are the mean water depth and phase-averaged water surface elevation.

The 2D RS formulation proposed by Longuet-Higgins and Stewart (1964) is expressed as follows:

$$
\left\{\begin{array}{c}
S_{x x}=E\left[\frac{C_{g}}{C}\left(\cos ^{2} \theta_{w}+1\right)-\frac{1}{2}\right]+\frac{k_{x} k_{x}}{k} \frac{C^{2}}{L} A_{R} \\
S_{y y}=E\left[\frac{C_{g}}{C}\left(\sin ^{2} \theta_{w}+1\right)-\frac{1}{2}\right]+\frac{k_{y} k_{y}}{k} \frac{C^{2}}{L} A_{R}, \\
S_{x y}=E \frac{C_{g}}{C} \cos \theta_{w} \sin \theta_{w}+\frac{k_{x} k_{y}}{k} \frac{C^{2}}{L} A_{R}
\end{array}\right.
$$

$E=\frac{1}{16} g H_{s}^{2}$

where $\left(S_{x x}, S_{y y}\right)$ in Eq. (4) represents the vertically-averaged RS in the ( $x$, $y$ ) plane, and $S_{x y}$ is the $x$ or $y$ component of the RS across the $y$ or $x$ plane. $E, H_{s}, L$, and $\theta_{w}$ refer to the wave energy, significant wave height, mean wave length and wave direction; $\left(k_{x}, k_{y}\right)$ are the wave number $\vec{k}$ in the $(x, y)$ plane with $k_{x}=|\vec{k}| \cos \theta_{w}$ and $k_{y}=|\vec{k}| \sin \theta_{w} ; C_{g}$ and $C$ are the wave group velocity and phase speed. The wave roller area $A_{R}$ is calculated based on the formulation proposed by Svendsen (1984).

A series of seminal papers by Mellor (2003, 2005; 2008; 2013; 2015) were published to propose and improve the depth-dependent 3D RS. The recent version (Mellor, 2015) implemented at FVCOM in this study was expressed as:

$\left\{\begin{array}{c}S_{x x}=k E\left(\frac{k_{x} k_{x}}{k^{2}} F_{C C} F_{C S}-F_{S S} F_{S C}\right)+\frac{E}{2 D} \mathfrak{\Im}(\varsigma)+\frac{k_{x} k_{x}}{k} \frac{C^{2}}{L} A_{R} R_{z} \\ S_{y y}=k E\left(\frac{k_{y} k_{y}}{k^{2}} F_{C C} F_{C S}-F_{S S} F_{S C}\right)+\frac{E}{2 D} \mathfrak{\Im}(\varsigma)+\frac{k_{y} k_{y}}{k} \frac{C^{2}}{L} A_{R} R_{z}, \\ S_{x y}=\frac{k_{x} k_{y}}{k^{2}} F_{C C} F_{C S}+\frac{k_{x} k_{y}}{k} \frac{C^{2}}{L} A_{R} R_{z}\end{array}\right.$

$\left\{\begin{array}{l}F_{S S}=\frac{\sinh k D(1+\varsigma)}{\sinh k D} \\ F_{S C}=\frac{\sinh k D(1+\varsigma)}{\cosh k D} \\ F_{C S}=\frac{\cosh k D(1+\varsigma)}{\sinh k D} \\ F_{C C}=\frac{\cosh k D(1+\varsigma)}{\cosh k D}\end{array}\right.$

$\mathfrak{J}(\varsigma)=\frac{\partial}{\partial \varsigma}\left(2 F_{C C} F_{S S}-F_{S S}^{2}\right)$

where $S_{x x}, S_{y y}$, and $S_{x y}$ in Eq. (6) are the 3D RS varying from the surface to bottom; the convenient definitions of $F_{S S}, F_{S C}, F_{C S}$, and $F_{C C}$ are the recurring depth-dependent functions. The sigma $\varsigma=\frac{z-\widehat{\eta}}{D}$ varies from -1 to 0 , with the corresponding $z$ increasing from the bottom level $-h$ to the mean water surface $\hat{\eta}$; the integration of $\mathfrak{\Im}(\varsigma)$ throughout the water column is $\int_{-1}^{0} \Im d \varsigma=1$. The vertical distribution function $R_{z}$ tailored for the wave-induced roller is derived from Svendsen et al. (2002).

\subsection{2. $3 D$ vortex-force formalism}

The vortex-force (VF) formalism applied to FVCOM is based on Uchiyama et al. (2010) and Kumar et al. (2012). The 3D Eulerian currents and Stokes drifts are defined as $(\vec{u}, w)$ and $\left(\overrightarrow{u^{s t}}, w^{S t}\right)$, and the current vector with an arrow on top means the horizontal velocities. The continuity and momentum balance applied with VF are written as: $\nabla_{\perp} \cdot \vec{u}+\frac{\partial w}{\partial z}=0$

$$
\begin{aligned}
& \frac{\partial \vec{u}}{\partial t}+\left(\vec{u} \cdot \nabla_{\perp}\right) \vec{u}+w \frac{\partial \vec{u}}{\partial z}+f \widehat{z} \times \vec{u}+\nabla_{\perp} \phi-\vec{F}+\vec{D}+\frac{\partial}{\partial z}\left(\overrightarrow{u w^{\prime}}-v \frac{\partial u}{\partial z}\right) \\
& =-\nabla_{\perp} \mathscr{K}+\vec{J}+\vec{F}^{w} \\
& \frac{\partial \phi}{\partial z}+\frac{g \rho}{\rho_{0}}=-\frac{\partial \mathscr{K}}{\partial z}+K
\end{aligned}
$$

where $f$ is the Coriolis parameter, $\phi$ is the dynamic pressure normalized by the density $\rho_{0}$. $(\vec{J}, K)$ represent the horizontal and vertical vortex forces:

$\vec{J}=-\widehat{z} \times \overrightarrow{u^{s t}}\left(\left(\widehat{z} \cdot \nabla_{\perp} \times \vec{u}\right)+f\right)-w^{s t} \frac{\partial \vec{u}}{\partial z}$,

$\mathrm{K}=\overrightarrow{u^{s t}} \cdot \frac{\partial \vec{u}}{\partial z}$

The Bernoulli head $\mathscr{K}$ is an adjustment of the mean pressure due to the presence of waves (Lane et al., 2007), which is expressed as:

$\mathscr{K}=\frac{\sigma H^{2}}{16 \cdot k \sinh ^{2} \mathscr{H}} \int_{-h}^{z} \frac{\partial^{2} \mathscr{V}}{\partial z^{2}} \sinh 2 k\left(z-z^{\prime}\right) d z^{\prime}$.

$\overrightarrow{F^{w}}$ is the wave-induced non-conservative forces (e.g., wave breaking and roller-induced acceleration). The 3D Stokes velocity $\left(\vec{u}^{S t}, w^{S t}\right)$ is expressed as follows:

$\overrightarrow{\mathrm{u}^{\mathrm{St}}}(\mathrm{z})=\frac{2 \mathrm{E}}{\mathrm{C}} \frac{\cosh 2|\overrightarrow{\mathbf{k}}|(\mathrm{z}+\mathrm{h})}{\sinh 2|\overrightarrow{\mathbf{k}}|(\mathrm{h}+\xi+\widehat{\xi})} \vec{k}$,

$w^{S t}=-\nabla_{\perp} \cdot \int_{-h}^{z} \overrightarrow{u^{S t}} d z$

The range of $z$ varies from $h(x) \leq z \leq \xi+\widehat{\xi}$, where $\xi$ and $\widehat{\xi}$ are the mean and wave-averaged sea levels.

The normalized vertical lengths $\mathscr{H}$ is given by:

$\mathscr{H}=|\vec{k}|(h+\xi+\widehat{\xi})=|\vec{k}| D$

The vertical Lagrangian velocity over the sigma space is given as:

$\omega^{l}=\left.\left[w^{l}-\left(\frac{\partial z}{\partial t}+\overrightarrow{u^{l}} \cdot \nabla_{\perp} z\right)\right]\right|_{s}$.

The relationships between $E, c,|\vec{k}|, \mathscr{H}, H_{r m s}$ (root-mean-square wave height), and $\sigma$ (intrinsic wave freqeucy) are:

$E=\frac{1}{8} g H_{r m s}^{2}, c=\frac{\sigma}{|\vec{k}|} \frac{\vec{k}}{|\vec{k}|}$ and $\sigma=\sqrt{g|\vec{k}| \tanh \mathscr{H} .}$

\subsection{Experimental design}

To compare the wave-induced effects between the 2D or 3D RS and the 3D VF-induced effects, numerical experiments were carried out in three classic systems including an idealized inlet, a planar beach, and a natural barred beach. Main components of the 3D VF utilized in the momentum balance include the horizontal and vertical advections of Stokes drifts, vortex force, wave-breaking and roller-induced accelerations, Stokes-Coriolis force, and Bernoulli head. To investigate the effects of wave-current interaction on coastal dynamics, simulations from the wave-only and circulation-only models in the first case of the idealized inlet were included. A more thorough discussion of the waveinduced 3D circulation and Stokes drifts between the 3D RS and VF 
methods was made in the following cases of the idealized planar and natural barred beaches in Section 3.

\subsection{Skill metrics}

Assessments of the model skill were based on the root-mean-squaredifference (RMSD), Pearson correlation coefficient (CC), and mean bias $(M B)$ :

$$
\begin{aligned}
& R M S D=\left(\frac{1}{N} \sum_{n=1}^{N}\left(\text { Model }_{n}-\text { Obs }_{n}\right)^{2}\right)^{1 / 2}, \\
& C C=\frac{\frac{1}{N} \sum_{n=1}^{N}\left(\text { Obs }_{n}-\overline{O b s}\right)\left(\text { Model }_{n}-\overline{\text { Model }}\right)}{\sigma_{\text {Obs }} \sigma_{\text {Model }_{n}}},
\end{aligned}
$$

$M B=\frac{1}{N} \sum_{n=1}^{N}\left(\right.$ Model $\left._{n}-\mathrm{Obs}_{n}\right)$,

where $\overline{\mathrm{Obs}}$ and $\overline{\mathrm{Model}}$ are the averaged values of observations $\left(\mathrm{Obs}_{n}\right)$ and model results $\left(\right.$ Model $_{n}$ ) in a sample of size $N . \sigma_{O s_{n}}$ and $\sigma_{\text {Model }_{n}}$ are the corresponding standard deviations. These metrics are widely used in ocean model evaluations (e.g., Liu et al., 2009). For correlation analysis, information about the degrees of freedom between group, within group, and in total ( $\mathrm{dfw}, \mathrm{dfb}$, and $\mathrm{df}$ ) together with the p-value are provided.

\section{Results and discussion}

\subsection{Application to an idealized tidal inlet}

The idealized tidal inlet covers a semi-enclosed rectangular basin with the width and length of 15.2 and $14 \mathrm{~km}$ (Fig. 1a), and the model settings are generally identical to Wu et al. (2011). The bathymetry is uniform in the $x$-direction, and it is $15 \mathrm{~m}$ along the open boundary at $y=$ $14 \mathrm{~km}$ and decreases southwardly to $4 \mathrm{~m}$ along the solid boundary at $y=$ 0 . The wall is oriented in $x$-direction with an extension of $200 \mathrm{~m}$ from $y$ $=6.6-6.8 \mathrm{~km}$. The inlet extends in the $x$ direction from 6.5 to $8.5 \mathrm{~km}$ with its width at $2 \mathrm{~km}$. The unstructured meshes include 10,508 elements and 5,467 nodes with a uniform size at $200 \mathrm{~m}$. The model comprises eight terrain-following sigma layers, which ensures the vertical resolutions of $0.5-1.9 \mathrm{~m}$ from shallow to deep waters. Open boundary conditions are specified by exerting the oscillating tidal level $\eta=$ $\cos \left(\frac{\pi}{6} t+\frac{\pi}{2}\right)$ (i.e., S2 tidal component with 1-m amplitude and 12-h period; the unit of $t$ is in hours) and incident waves with 1-m significant wave height (SWH) and 10-s peak period. The external time step is $0.25 \mathrm{~s}$ with a mode-split ratio of 20 , and the wave calculation and coupling steps are taken at every $5 \mathrm{~s}$. Detailed model settings are listed in Table 1.

Given that spatial wave distributions from the three coupled models are similar, simulated SWH only from the 3D RS model at ebb and flood phases $(t=26$ and $31 \mathrm{~h})$ are shown in Fig. 2a. Waves propagated from the open boundary with a significant reduction as they passed through the inlet (e.g., from 1.5/0.75 to $0.45 \mathrm{~m}$ at the ebb/flood phase). This finding is consistent with that of Wu et al. (2011) and a realistic lagoon-inlet-coastal ocean system of Mao and Xia (2018). Spatial variations of SWH between the ebb and flood phases near the inlet region were caused by the current-induced Doppler shift (Olabarrieta et al., 2011), which transported the wave energy northwardly and southwardly, respectively (Fig. 2b). The enhanced/reduced SWH by the opposing/following currents near the inlet found in this study are consistent with an idealized case conducted by Qi et al. (2009), and realistic shelf seas (Tolman, 1990) and a semi-enclosed basin (Benetazzo et al., 2013).

To examine performances of the implemented 3D RS and VF formulations in model simulations, time series of simulated SWH and y-
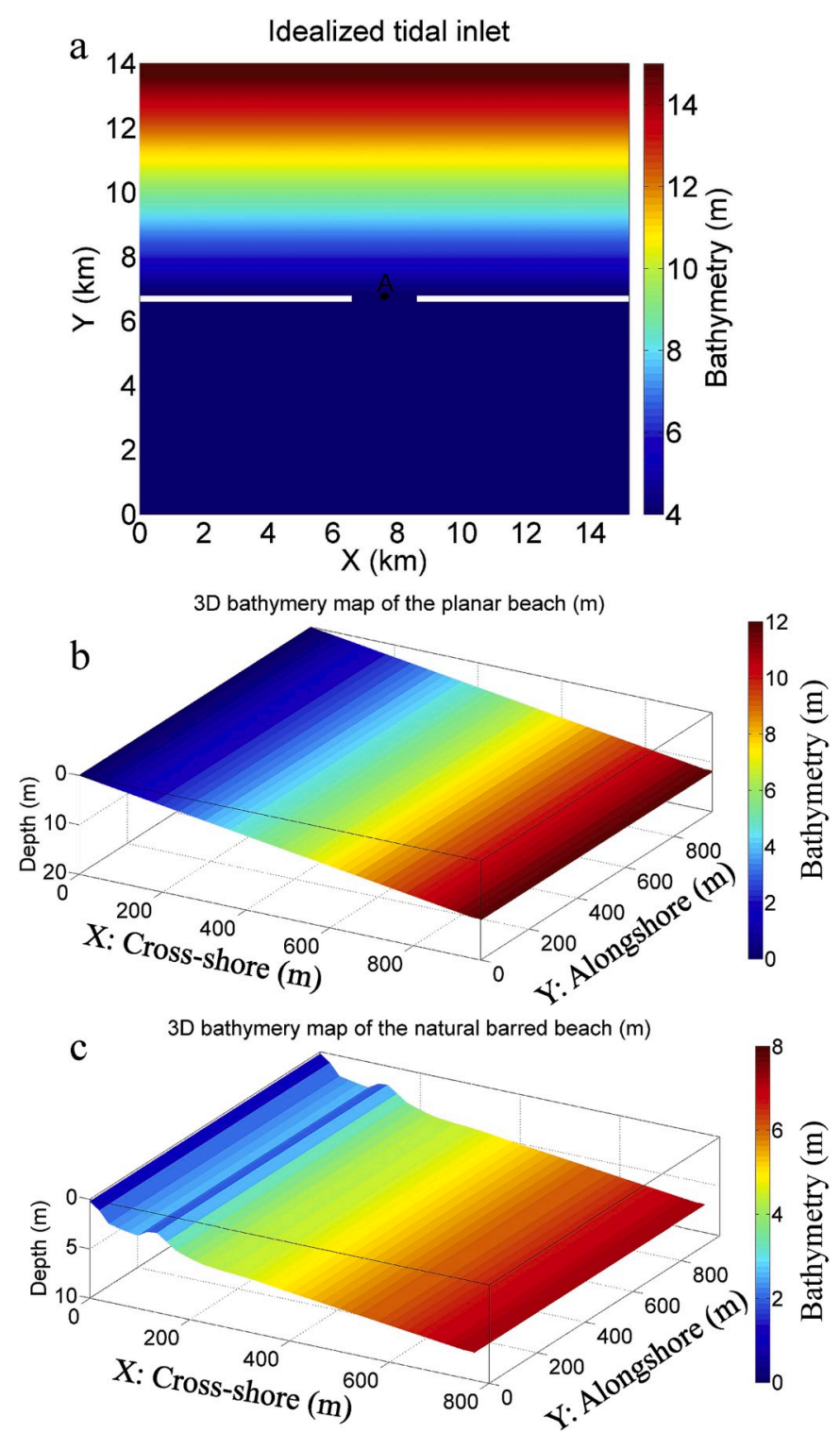

Fig. 1. Model domain and bathymetry of the (a) idealized inlet, (b) planar beach and (c) natural barred beach. Point $\mathrm{A}$ is located at the middle of the inlet.

component surface current velocity in the middle inlet (i.e., point A in Fig. 1a) were compared with those from Wu et al. (2011) (Fig. 3). By including wave-current interaction, nonlinear modulations of surface currents on SWH were detected periodically during the ebb-flood cycles. Overall, similar SWH $(C C=1.00$ and $R M S D=0.03 \mathrm{~m} ; \mathrm{dfw}=96, \mathrm{dfb}=$ $1, \mathrm{df}=97$, and p-value $<0.05)$ and y-component of surface current velocity $(C C=1.00$ and $R M S D=0.08 \mathrm{~m} / \mathrm{s} ; \mathrm{dfw}=96, \mathrm{dfb}=1, \mathrm{df}=97$, and p-value $<0.05$ ) were produced from the 3D RS and VF models. Although the 3D RS method showed larger variations of SWH and $\mathrm{y}$-component surface current velocity $(R M S D=0.05 \mathrm{~m}$ and $0.24 \mathrm{~m} / \mathrm{s}$ ) relative to those from Wu et al. (2011), temporal trends of both variables were captured reasonably well $(C C=1.00$; $\mathrm{dfw}=96, \mathrm{dfb}=1, \mathrm{df}=97$, and p-value $<0.05$ ). It should be noted that time series of SWH and y-component surface current velocity in the middle inlet from the 2D RS method were almost identical to those from the 3D RS model, and thus their simulations were not shown.

After having tested the model performance, various wave-current coupled models were compared for the wave-induced depth-averaged circulation (Fig. 4). Spatial variability of the wave-induced depthaveraged circulation between the ebb and flood phases from the 3D RS/ 
Table 1

Model parameters and settings of the idealized tidal inlet, planar beach, and natural barred beach.

\begin{tabular}{|c|c|c|}
\hline Cases & Parameters & Settings \\
\hline \multirow[t]{7}{*}{$\begin{array}{l}\text { Idealized } \\
\text { tidal inlet }\end{array}$} & $\begin{array}{l}\text { Model length and } \\
\text { width }\end{array}$ & $15 \mathrm{~km}$ and $14 \mathrm{~km}$ \\
\hline & $\begin{array}{l}\text { Inlet length and } \\
\text { width }\end{array}$ & $200 \mathrm{~m}$ and $2 \mathrm{~km}$ \\
\hline & $\begin{array}{l}\text { Elements, nodes, } \\
\text { and sigma layers }\end{array}$ & $10,508,5,467$, and 8 \\
\hline & $\begin{array}{l}\text { Open boundary } \\
\text { conditions }\end{array}$ & $\begin{array}{l}\text { Tide: } 1-\mathrm{m} \text { amplitude and } 12 \text {-h period; } \\
\text { Wave: } 1 \text {-m significant wave height and } 10 \text { - } \\
\text { s peak period, and } 270^{\circ} \text { mean direction }\end{array}$ \\
\hline & $\begin{array}{l}\text { External and } \\
\text { internal time steps }\end{array}$ & $0.25 \mathrm{~s}$ and $5 \mathrm{~s}$ \\
\hline & Grid size & $200 \times 200 \mathrm{~m}$ in $\mathrm{X}$ and $\mathrm{Y}$ directions \\
\hline & Simulation period & $2 \mathrm{~d}$ \\
\hline \multirow[t]{6}{*}{ Planar beach } & $\begin{array}{l}\text { Model length and } \\
\text { width }\end{array}$ & $960 \mathrm{~m}$ in $\mathrm{X}$ and $\mathrm{Y}$ directions \\
\hline & $\begin{array}{l}\text { Element, node, and } \\
\text { sigma layer }\end{array}$ & 4608,2401 , and 20 \\
\hline & $\begin{array}{l}\text { Open boundary } \\
\text { condition }\end{array}$ & $\begin{array}{l}\text { Wave: } 2 \text {-m significant height, } 10 \text {-s peak } \\
\text { period, and } 170^{\circ} \text { mean direction }\end{array}$ \\
\hline & $\begin{array}{l}\text { External and } \\
\text { internal time steps }\end{array}$ & $0.05 \mathrm{~s}$ and $0.25 \mathrm{~s}$ \\
\hline & Grid size & $20 \times 20 \mathrm{~m}$ in $\mathrm{X}$ and $\mathrm{Y}$ directions \\
\hline & Simulation period & $8 \mathrm{~h}$ \\
\hline \multirow{7}{*}{$\begin{array}{l}\text { Natural } \\
\text { barred } \\
\text { beach }\end{array}$} & $\begin{array}{l}\text { Model length and } \\
\text { width }\end{array}$ & $\begin{array}{l}770 \text { and } 962.5 \mathrm{~m} \text { in cross-shore and } \\
\text { alongshore directions }\end{array}$ \\
\hline & $\begin{array}{l}\text { Element, node, and } \\
\text { sigma layer }\end{array}$ & $4,000,2,091$, and 20 \\
\hline & $\begin{array}{l}\text { Open boundary } \\
\text { condition }\end{array}$ & $\begin{array}{l}\text { Offshore tidal elevation } 0.7 \mathrm{~m} \\
\text { Wave: } 1.6-\mathrm{m} \text { root-mean-square height, 6-s } \\
\text { peak period, and } 193^{\circ} \text { mean direction }\end{array}$ \\
\hline & $\begin{array}{l}\text { External and } \\
\text { internal time steps }\end{array}$ & $0.05 \mathrm{~s}$ and $0.5 \mathrm{~s}$ \\
\hline & Grid size & $19.25 \times 19.25 \mathrm{~m}$ in $\mathrm{X}$ and $\mathrm{Y}$ directions \\
\hline & $\begin{array}{l}\text { Simulation period } \\
\text { Wind stress in }(x, y) \\
\text { direction }\end{array}$ & $\begin{array}{l}8 \mathrm{~h} \\
(-0.2532,-0.1456) \mathrm{Pa}\end{array}$ \\
\hline & Coriolis frequency $f$ & $8.5695 \times 10^{-5} \mathrm{~s}^{-1}$ \\
\hline
\end{tabular}

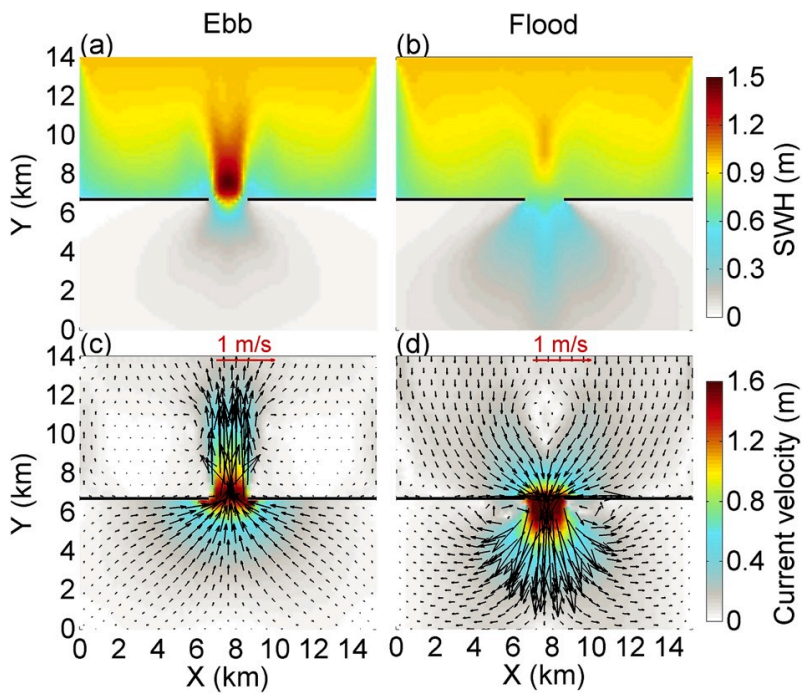

Fig. 2. (a)-(b) Significant wave height (SWH) and (c)-(d) depth-averaged current velocity fields produced from the 3D RS model at (a) and (c) ebb and (b) and (d) flood phases.

VF was caused by spatial inhomogeneities of the corresponding wave fields, which resulted originally from the current-induced Doppler shift (Fig. 2). Specifically, the 3D RS produced discernable longshore currents around $0.1 \mathrm{~m} / \mathrm{s}$ near the barriers and lateral boundaries, forming a pair of circular cells with sizes of $3-4 \mathrm{~km}$ near the corner. At the ebb phase, the $3 \mathrm{D} V F$ generated strong currents that passed through the inlet with intensities up to $0.2 \mathrm{~m} / \mathrm{s}$. At the flood phase, this type of 3D VF-induced currents shifted approximately 4-km northward with additional diverging currents at $\sim 0.1 \mathrm{~m} / \mathrm{s}$ along the $x$ direction. As a result of weak wave activities behind the barriers and away from the inlet region (e.g., $<0.2 \mathrm{~m}$ in Fig. $2 \mathrm{a}$ and b), wave-induced currents were correspondingly negligible (e.g., $<0.02 \mathrm{~m} / \mathrm{s}$ in Fig. $4 \mathrm{a}-\mathrm{d}$ ) using both methods.

Recently, Mellor (2016) provided a method to compare the wave-induced effects between the 3D RS and VF theories, and it is evaluated in the idealized inlet case (Fig. 5). It was mostly between -5 and -3 with the largest one at -1 in front of the inlet at the flood phase, confirming that "the ratio of the vortex force to the wave radiation stress term is of order $(k a)^{2}\left(\approx 10^{-2}\right)$ so that the vortex force term can be discarded." The vortex-force mentioned in Mellor (2016) denotes the interaction of horizontal Stokes drifts with the curl of horizontal Eulerian velocities $\left(u^{s} \times \omega_{0}=u^{s} \times\left(\nabla \times \overrightarrow{v_{0}}\right)\right)$, in which non-conservative forcing such as wave breaking and roller-induced accelerations are not considered (Uchiyama et al., 2010; Kumar et al., 2012). By excluding the vortex-force from the 3D VF, wave-induced currents relative to those from the full $3 \mathrm{D} \mathrm{VF}$ simulations changed $\sim 0.05 \mathrm{~m} / \mathrm{s}$ in the areas with strong wave-current interaction (Fig. 4c-f). However, the breaking and roller-induced circulation reached a variation of $\sim 0.15 \mathrm{~m} / \mathrm{s}$ in front of the inlet when this term was excluded from the 3D VF (Fig. 4c-d and $\mathrm{g}-\mathrm{h}$ ). This phenomenon suggests that the non-conservative forcing is primarily responsible for the wave-induced currents in the idealized inlet case, followed by the conservative vortex-force effect. Therefore, both forces were included in the 3D VF theory applied to the current modeling system. The 2D RS-induced depth-averaged circulation was very similar to those from the 3D RS method and thus was not shown.

To investigate the vertical variability, the wave-induced circulation in the surface and bottom layers from the 3D RS and VF methods at the ebb phase is shown in Fig. 6 . As expected, both produced stronger waveinduced currents in the surface layer than those in the bottom layer (up to 0.3 versus $<0.15 \mathrm{~m} / \mathrm{s}$ near the inlet), which can be attributed to the vertically decreasing functions of the wave forcing. Specifically, the type III vertical distribution function $f^{b}(z) \propto \cosh k_{b}(z+h)$ with a choice of $k_{b}=$ $2|\overrightarrow{\mathrm{k}}|$ was selected for the wave breaking and roller-induced effects in the 3D VF method (Eq. (53) in Uchiyama et al., 2010). In the 3D RS method, the vertically decreasing hyperbolic sine and cosine functions (i.e. $\sinh k D(1+\varsigma)$ and $\cosh k D(1+\varsigma)$ were included in Eqs. (6)-(8). In the surface layer both methods produced intensive currents in front of the inlet, and the 3D RS and VF-induced variations reached 0.15 and 0.3 $\mathrm{m} / \mathrm{s}$, respectively. Additionally, the 3D RS generated discernable longshore currents and circular cells with intensities up to $0.1 \mathrm{~m} / \mathrm{s}$ near the barriers and lateral sides. In the bottom layers, both the 3D RS and VF-induced currents resembled their surface distributions, but the intensities were reduced by about half. The 2D RS results were generally identical to 3D RS simulations, with the wave-induced circulation being slightly weaker in the surface layer (not shown). To further understand the variability of wave-induced circulation between the two methods, the modeling systems were applied to a planar beach forced by obliquely incident waves along the open boundary.

\subsection{Model comparisons for a planar beach}

The planar beach covers a gently sloping square basin with a side length of $960 \mathrm{~m}$ (Fig. 1b), and the bathymetry is uniform in the alongshore direction and linearly increases from 0 to $12 \mathrm{~m}$ in the cross-shore direction at a constant slope of 1:80. The unstructured meshes in the horizontal space include 4,608 elements and 2,401 nodes, with a uniform size of $20 \mathrm{~m}$. In the vertical coordinate, 20 terrain-following sigma layers were applied with a maximum resolution of $0.6 \mathrm{~m}$. Along the open 


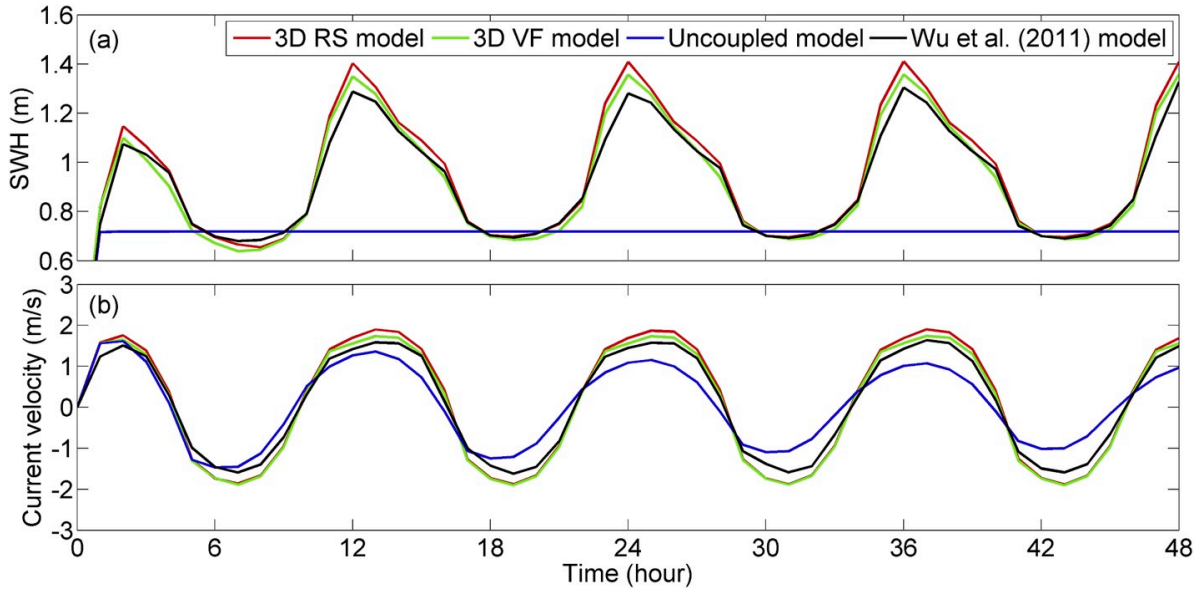

Fig. 3. Time series of SWH and surface current velocity at point A in the middle of the inlet produced from the 3D RS, VF, uncoupled, and Wu et al. (2011)'s models.

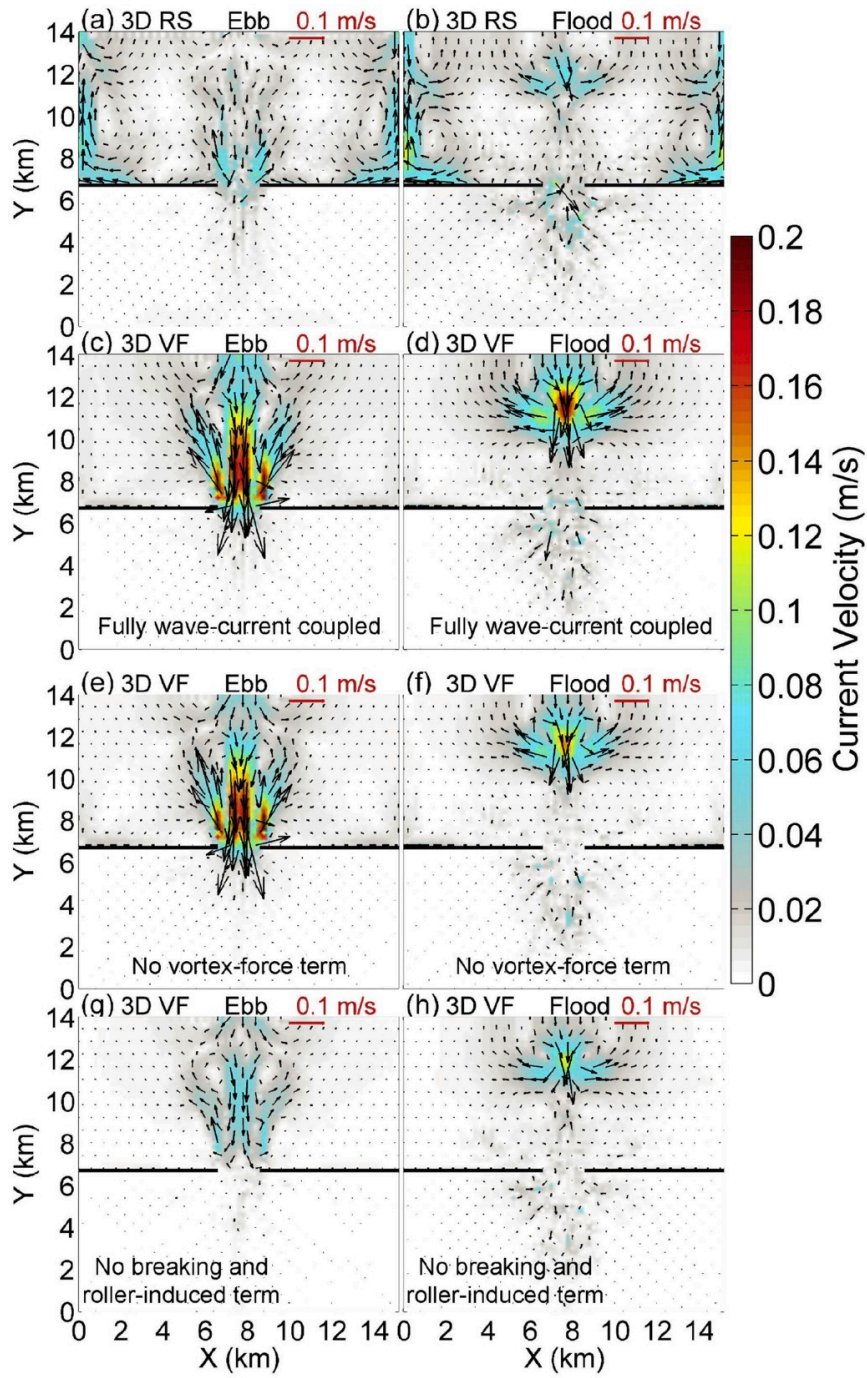

Fig. 4. Wave-induced depth-averaged circulation from (a)-(b) 3D RS, (c)-(d) $\mathrm{VF}$, and the VF method (e)-(f) without vortex-force and (g)-(h) breaking and roller-induced momentum flux at ebb and flood phases, respectively.

boundary layer, the model was forced by waves with 2-m SWH, 10-s peak period, and $10^{\circ}$ oblique angle relative to the shore-normal direction (Table 1). Spectral wave conditions were specified by the traditional

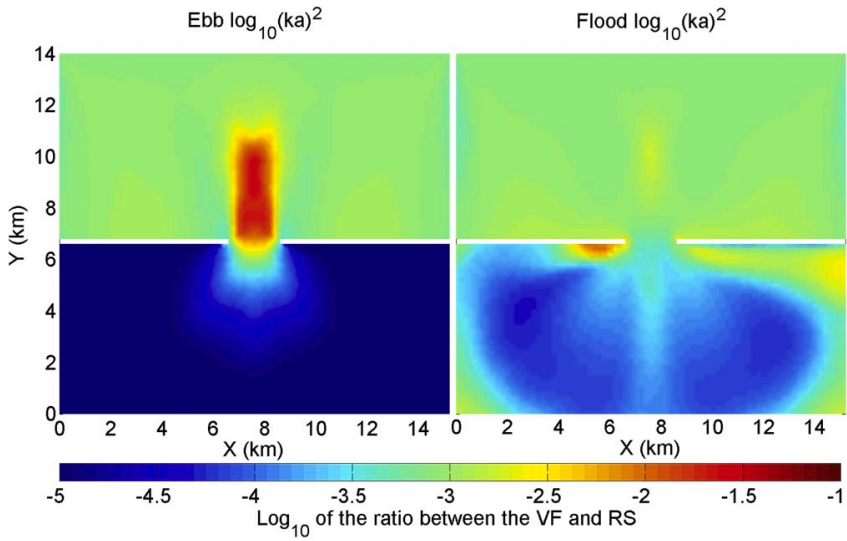

Fig. 5. Spatial distributions of the base-10 logarithm to the ratio between the VF and RS representations based on the formulation suggested by Mellor (2016).

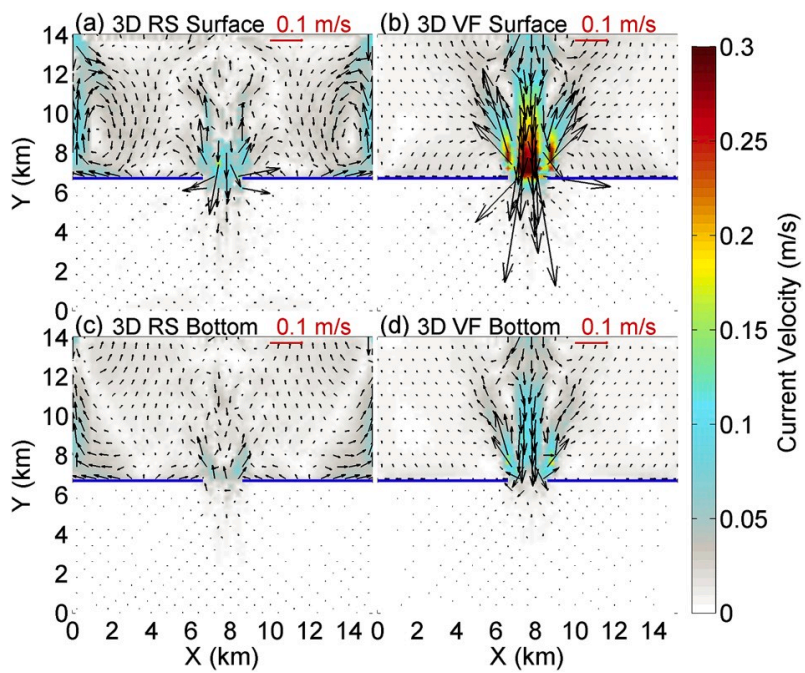

Fig. 6. Wave-induced circulation from the (a) and (c) 3D RS and (b) and (d) VF methods at (a)-(b) surface and (c)-(d) bottom layers at the ebb phase.

JONSWAP spectrum. Numerical runs were conducted for $8 \mathrm{~h}$ to obtain stable results, and the external time step was set at $0.05 \mathrm{~s}$ with a modesplit ratio of 5 . The wave-current coupling process was executed at 
every $0.5 \mathrm{~s}$ and the spin-up period was $1.5 \mathrm{~min}$. The physical settings are basically identical to those of Uchiyama et al. (2010), in which the air-sea transfers of heat flux and winds are not considered.

Wave-induced Eulerian currents in the cross-shore sections from the 3D RS and VF representations are shown in Fig. 7. The two-layer flow pattern (i.e., surface onshore and bottom undertow currents) with an intensity up to $0.5 \mathrm{~m} / \mathrm{s}$ was characterized inside the surf zone at $\mathrm{x}<500$ $\mathrm{m}$, consistent with previous field observations (Ting and Kirby, 1994) and numerical results (Kumar et al., 2012). By applying the 3D RS method, this type of two-way circulation shifted towards offshore regions $(x>300 \mathrm{~m})$ and the intensity of the cross-shore current was reduced to less than $0.2 \mathrm{~m} / \mathrm{s}$. This phenomenon was caused by the unrealistic offshore currents generated by the vertical RS flux adjacent to the shore (Kumar et al., 2011; Moghimi et al., 2013). A direct comparison of the wave-induced circulation between the paired methods were shown in this study. Most recently, Ji et al. (2019) implemented the modified 3D RS theory (e.g., the vertical RS flux term $\frac{E}{2 D} \mathfrak{I}(\varsigma)$ in Eq. (8), see Ji et al., 2017) in FVCOM for nearshore applications, and showed an improved performance in simulating wave-induced currents. It is worthwhile in future endeavors to compare this modeling system with the modified 3D RS theory, but it is beyond the scope of this study.

Both models successfully produced strong longshore currents within the surf zone, which were up to 0.7 and $1 \mathrm{~m} / \mathrm{s}$ from the 3D RS and VF, respectively (Fig. $7 \mathrm{~d}$ and e). The presence of the intensified longshore currents detected within the surf zone and near the coast in this study was supported by previous results (Uchiyama et al., 2010; Kumar et al., 2012; Zheng et al., 2017; Ji et al., 2019). This fact suggests that our implementations of both theories are effective and satisfactory in simulating the wave-induced longshore currents in the case of the planar beach. Additional numerical runs indicate that the breaking and roller-induced accelerations are primarily responsible for the wave-induced coastal circulation in the nearshore regions (Fig. $7 \mathrm{c}$ and $\mathrm{f}$ ). The maximum wave breaking point was located at $x \sim 100 \mathrm{~m}$, where the strongest longshore currents were detected and gradually became weaker inshore. This 3D circulation pattern induced by the breaking and roller-induced accelerations resembles previous modeling studies from Uchiyama et al. (2010) and Kumar et al. (2012). It can be concluded that the breaking and roller-induced longshore currents and associated vertically recirculating cross-shore currents (e.g., shoreward surface flow and the compensated near-bottom seaward undertow) are mainly caused by the surface-intensified breaker acceleration.

In addition to comparing the Eulerian currents, wave-induced horizontal Stokes drifts from the paired methods are shown in Fig. 8. It was not surprising that spatial distributions of horizontal Stokes drifts were almost identical between both wave-current coupled models. Because offshore waves mainly propagated toward the shoreline in a shorenormal direction (e.g., $\left.\left|k_{x}\right| \gg\left|k_{y}\right|\right)$, the cross-shore component of the Stokes drift $\overrightarrow{u^{S t}}(z)=\frac{2 E}{C} \frac{\cosh 2|\vec{k}|(z+h)}{\sinh 2 k D} \vec{k}$ was approximately one order of magnitude greater than the alongshore component $(O(0.1)$ versus $O$ $(0.01) \mathrm{m} / \mathrm{s})$. As a result of the shoaling process in the shallower region, the wave number and corresponding Stokes drift were enhanced gradually to $-0.16 \mathrm{~m} / \mathrm{s}$ before showing a decreasing trend by the significant depth-induced wave breaking. The monotonic decreasing of horizontal Stokes drifts from the surface to bottom layers was highly associated with the hyperbolic function in the expression of the Stokes drift, following a $\cosh 2|\vec{k}|(z+h)$ distribution in the vertical space from both methods. Consequently, the strongest Stokes drift was near the surface layer and the weakest one was close to the bottom, consistent with previous works of Kumar et al. (2012) and Zheng et al. (2017).

\subsection{Sandbar DUCK'94 experiment}

After having successfully applied the wave-current coupled models to the idealized inlet and slope cases, wave-induced effects in a natural barred beach were examined and discussed thoroughly in this subsection. The DUCK's 94 experiment was conducted on October 12, 1994 under a stormy condition (Newberger and Allen, 2007). The cross-shore and alongshore extension of the model domain was $770 \mathrm{~m}$ by $962.5 \mathrm{~m}$ in the $x$ and $y$ directions, and the horizontal grid size was spatially uniform at $19.25 \mathrm{~m}$. Water depths were uniform in the alongshore direction, and varied from $7.26 \mathrm{~m}$ along the offshore boundary to zero near the shoreline (Fig. 1c). A constant tidal elevation of $0.7 \mathrm{~m}$ was exerted at the offshore boundary, and the sandbar was situated at $x \sim 130 \mathrm{~m}$ from the shoreline. Along the open boundary, the incident waves were specified by the JONSWAP spectrum at 1.6-m root-mean-square height $H_{r m s}$ and 6 -s peak period, which propagated at a mean direction of $193^{\circ}$. Given that all forcing factors that included winds, waves and tides were averaged over the measurement period, they were kept constant in the numerical model (Table 1 ). The external time step was set at $0.05 \mathrm{~s}$ with a mode-splitting ratio of 10; wave calculations and coupling processes were the same and taken at every $0.5 \mathrm{~s}$. The spin-up period is $3 \mathrm{~min}$ and stable solutions were acquired after 8-h simulations.

The observational data used to validate model performances in the natural sandbar experiment were collected from various resources. The current velocity and wave height data at 13 and 15 cross-shore sites
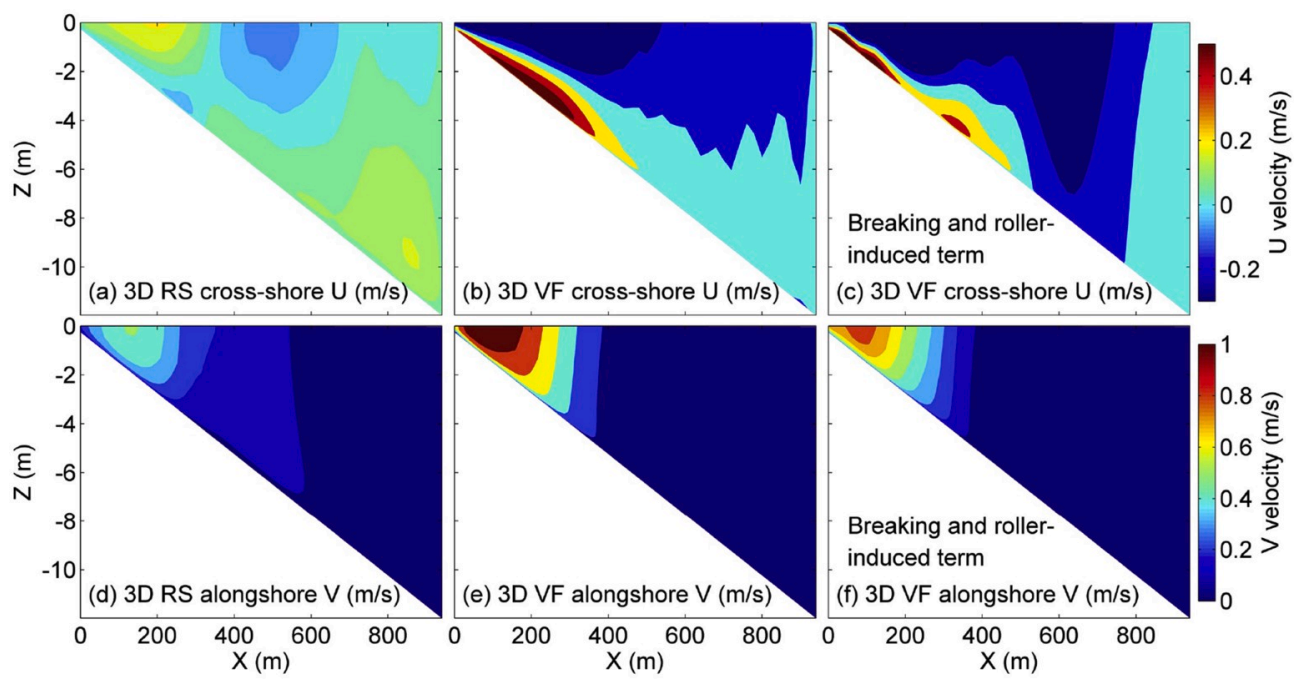

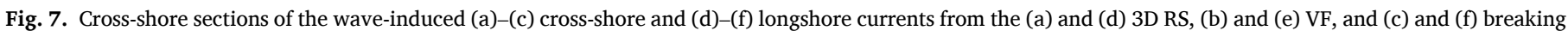
and roller-induced acceleration in the 3D VF method. 

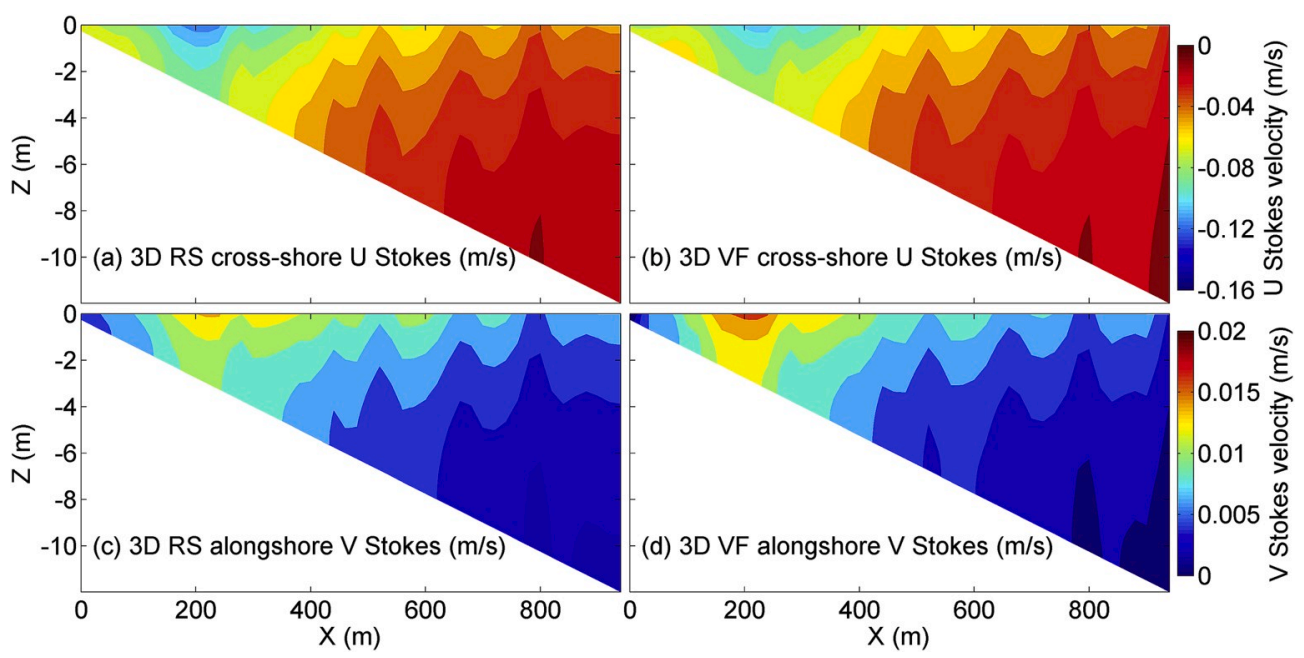

Fig. 8. Cross-shore sections of the wave-induced (a) and (b) cross-shore and (c) and (d) longshore Stokes drifts from the (a) and (c) 3D RS and (b) and (d) VF methods.

(Fig. 9a-e and Table 2) were provided by Dr. S. Elgar, and additional cross-shore current profiles near the complex sandbar region (Fig. $9 \mathrm{f}$ and g) were shared by Dr. S. Moghimi. Detailed descriptions of the DUCK' 94 measurements can be found in Elgar et al. (1997) and Feddersen et al. (1998). The simulated $H_{r m s}$ using both wave-current models (Fig. 9a) captured spatial variations of observed values (e.g., significant reductions near the sandbar and close to the shore) satisfactorily with $C C \geq 0.97(\mathrm{dfw}=28, \mathrm{dfb}=1, \mathrm{df}=29$, and $\mathrm{p}$-value $<0.05$ ) and $R M S D \leq$ $0.19 \mathrm{~m}$. Because current velocities in Fig. 9b were mainly measured near the bottom, the positive cross-shore $U$ indicated offshore undertows. The $3 \mathrm{D} V \mathrm{~V}$ reproduced the bottom $U$ slightly better than that simulated from the 3D RS, while the latter method outperformed the former one marginally regarding the longshore current $V$. For the vertical profile of $U$ and $V$ (Fig. $9 \mathrm{~d}$ and e), the RMSD from the 3D RS method were 0.04 and $0.2 \mathrm{~m} / \mathrm{s}$ respectively, while they were 0.07 and $0.2 \mathrm{~m} / \mathrm{s}$ from the $3 \mathrm{D} \mathrm{VF}$ model. With a closer examination of the $3 \mathrm{D}$ circulation profile near the complex sandbar region (Fig. 9f and g), it was found that both methods followed the vertically decreasing trend of longshore currents from the surface to bottom layers reasonably well. Additionally, the 3D RS method showed a smaller deviation of longshore currents from observations than that in the $3 \mathrm{D}$ VF method ( $M B$ of 0.21 versus $0.28 \mathrm{~m} / \mathrm{s}$ ), especially in front of the sandbar (Table 3). For the cross-shore current $U$, the 3D VF representation was in better agreement with observations than the 3D RS model (CC of 0.87 versus 0.33 ; $\mathrm{dfw}=12$, $\mathrm{dfb}=1$, and $\mathrm{df}$ $=13$; $\mathrm{p}$-value of 0.03 versus 0.20 ). From the perspective of nearshore dynamics, the 3D VF reproduced vertical variations of cross-shore currents (e.g., surface onshore and bottom undertow) more physically reasonable than the 3D RS method (Fig. 9f). This finding consists with previous studies of Kumar et al. (2011) and Moghimi et al. (2013), suggesting that additional improvement on the vertical RS flux term is a worthwhile future endeavor (Ji et al., 2017).

To further compare the 3D circulation structures yielded from the
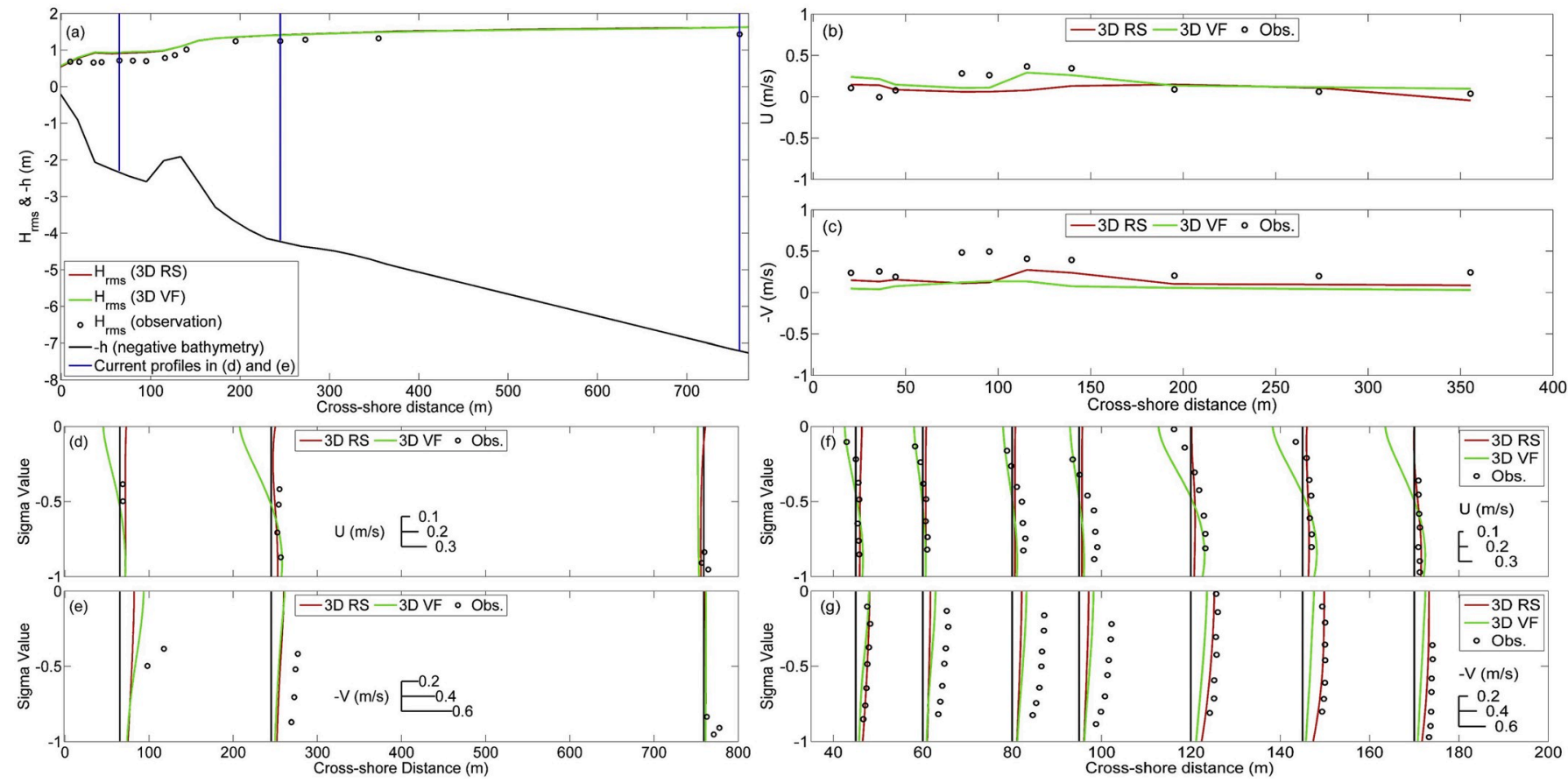

Fig. 9. Cross-shore sections of (a) wave height $H_{r m s}$, (b) cross-shore $U$, (c) longshore $-V$, and profiles of (d) and (f) $U$ and (e) and (g) $-V$ from observations and simulations. 
Table 2

Geographic information of the observed DUCK'94 data.

\begin{tabular}{|c|c|c|c|c|}
\hline \multicolumn{3}{|c|}{ Current velocity } & \multicolumn{2}{|c|}{ Significant wave height } \\
\hline Obs. station & Cross-shore distance from shoreline (m) & Vertical distance relative to mean sea level $(\mathrm{cm})$ & Obs. station & Cross-shore distance from shoreline $(\mathrm{m})$ \\
\hline Uv03 & 20 & -77 & P02 & 10 \\
\hline Uv04 & 36 & -141.5 & P03 & 20 \\
\hline Uv05 & 44 & -161 & P04 & 36 \\
\hline Uv21 & 65 & -43 & P05 & 45 \\
\hline Uv22 & 65 & -78 & P23 & 65 \\
\hline Uv12 & 80 & -168.6 & $\mathrm{P} 12$ & 80 \\
\hline Uv13 & 95 & -152 & P13 & 95 \\
\hline Uv14 & 116 & -115.5 & P14 & 116 \\
\hline Uv15 & 140 & -173 & P16 & 127 \\
\hline Uv17 & 195 & -311 & P15 & 140 \\
\hline Uv41 & 245 & -132 & P17 & 195 \\
\hline Uv42 & 245 & -182 & P45 & 245 \\
\hline Uv43 & 245 & -272 & P18 & 273 \\
\hline Uv44 & 245 & -352 & P19 & 355 \\
\hline Uv18 & 273 & -381 & P87 & 759 \\
\hline Uv19 & 355 & -476 & & \\
\hline Uv85 & 759 & -632 & & \\
\hline Uv86 & 759 & -692 & & \\
\hline Uv87 & 759 & -729 & & \\
\hline
\end{tabular}

Table 3

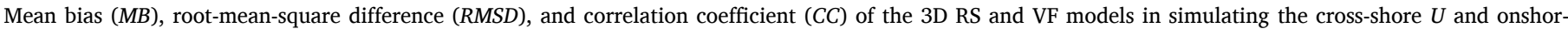
e-offshore $V$ for the DUCK' 94 case at seven sections near the sandbar.

\begin{tabular}{|c|c|c|c|c|c|c|c|c|c|c|}
\hline & & Location & S1 & S2 & S3 & S4 & S5 & S6 & S7 & Mean \\
\hline $3 \mathrm{D}$ & $\mathrm{U}$ & $\mathrm{MB}(\mathrm{m} / \mathrm{s})$ & 0.08 & 0.05 & -0.08 & -0.15 & -0.04 & 0.00 & 0.00 & -0.02 \\
\hline \multirow[t]{5}{*}{ RS } & & RMSD (m/s) & 0.14 & 0.10 & 0.16 & 0.24 & 0.22 & 0.10 & 0.04 & 0.14 \\
\hline & & $\mathrm{CC}$ & -0.89 & -0.18 & 0.35 & 0.69 & 0.96 & 0.76 & 0.62 & 0.33 \\
\hline & $\mathrm{V}$ & $\mathrm{MB}(\mathrm{m} / \mathrm{s})$ & 0.00 & 0.33 & 0.45 & 0.43 & 0.09 & 0.05 & 0.12 & 0.21 \\
\hline & & $\mathrm{RMSD}(\mathrm{m} / \mathrm{s})$ & 0.02 & 0.33 & 0.46 & 0.44 & 0.10 & 0.06 & 0.12 & 0.22 \\
\hline & & $\mathrm{CC}$ & 0.88 & 0.98 & 0.96 & 0.96 & 0.90 & 0.46 & 0.96 & 0.87 \\
\hline $3 \mathrm{D}$ & $\mathrm{U}$ & $\mathrm{MB}(\mathrm{m} / \mathrm{s})$ & -0.02 & -0.05 & -0.14 & -0.2 & -0.23 & -0.17 & 0.00 & -0.12 \\
\hline \multirow[t]{5}{*}{$\mathrm{VF}$} & & $\mathrm{RMSD}(\mathrm{m} / \mathrm{s})$ & 0.09 & 0.06 & 0.16 & 0.23 & 0.28 & 0.29 & 0.14 & 0.18 \\
\hline & & CC & 0.78 & 0.95 & 0.97 & 0.97 & 0.97 & 0.85 & 0.63 & 0.87 \\
\hline & $\mathrm{V}$ & $\mathrm{MB}(\mathrm{m} / \mathrm{s})$ & 0.07 & 0.28 & 0.39 & 0.38 & 0.26 & 0.30 & 0.25 & 0.28 \\
\hline & & $\mathrm{RMSD}(\mathrm{m} / \mathrm{s})$ & 0.08 & 0.28 & 0.39 & 0.38 & 0.27 & 0.31 & 0.25 & 0.28 \\
\hline & & $\mathrm{CC}$ & 0.80 & 0.96 & 0.96 & 0.96 & 0.86 & 0.20 & 0.92 & 0.81 \\
\hline
\end{tabular}

paired modeling systems, vertical profiles of the Eulerian currents in the cross-shore direction are shown in Fig. 10. In the 3D VF simulations, strong surface onshore flows up to $0.4 \mathrm{~m} / \mathrm{s}$ and bottom undertow currents up to $0.3 \mathrm{~m} / \mathrm{s}$ were reasonably reproduced (Fig. 10b). Additionally, the offshore upward and coastal downward flows up to $0.01 \mathrm{~m} / \mathrm{s}$ within the surf zone region were formed, which led to the formation of a circular cell in the nearshore (Fig. 10h). By contrast, 3D RS-induced offshore currents close to the shoreline with velocities $0-0.1 \mathrm{~m} / \mathrm{s}$ were detected throughout the water column (Fig. 10a). Moreover, the vertical upward and downward movement cycle was weak $(<0.005 \mathrm{~m} / \mathrm{s})$ and located further away from the shoreline (e.g., in front of the sandbar, see Fig. 10g), which was similar to the case of the idealized planar beach. However, both methods showed intensified bottom undertows over the sandbar's crest, consistent with previous results of Kumar et al. (2012) and Moghimi et al. (2013). This finding demonstrates that both models reproduce the two-way flow pattern satisfactorily, yet additional efforts are required to further reduce the 3D RS-generated unrealistic offshore currents near the shoreline. Locations of the maximum longshore current velocities (e.g., 0.5 and $0.4 \mathrm{~m} / \mathrm{s}$ in the 3D RS and VF) were near the sandbar region and close to the shoreline (Fig. 10d and e). The location and intensity of the locally maximum longshore currents differed slightly from those of Kumar et al. (2012), presumably due to different settings in the tuned parameters of roller-induced momentum transport (e.g., the fraction of surface rollers in broken waves). Further numerical experiments indicate that the breaking and roller-induced accelerations are the mainly forcing term of the 3D VF method in this natural barred case (Fig. 10c, f, and i), and the maximum longshore currents due to wave breaking were detected near the sandbar. Additionally, the cross-shore undertow currents due to the breaking and roller-induced accelerations were increased near the coast and the crest of the sandbar (Fig. 10c). These surface onshore and bottom undertow flows further formed an anticlockwise circulation cell pattern (Fig. 10c and i), which was consistent with previous modeling studies (Uchiyama et al., 2010; Kumar et al., 2012).

Wave-induced horizontal Stokes drifts from both models were quite similar (Fig. 11a-d), which followed the hyperbolic cosh2 $|\vec{k}|(z+h)$ function. Consequently, the strongest and weakest ones appeared near the surface bottom layers, respectively. Moreover, the nearly shorenormal directed incoming waves generated the Stokes drift up to 0.2 $\mathrm{m} / \mathrm{s}$ in the onshore direction, and less than $0.03 \mathrm{~m} / \mathrm{s}$ in the alongshore direction. These abovementioned spatial variations of horizontal Stokes drift in the natural sandbar case are supported by previous works of Kumar et al. (2012) and Zheng et al. (2017). It should be noted that the magnitude of vertical Stokes drift calculated in the 3D VF theory was comparable to its longshore component at $O(0.01) \mathrm{m} / \mathrm{s}$ (Fig. $11 \mathrm{~d}$ and f), while it is zero over the entire domain in the 3D RS method. The only region with nearly zero value of vertical Stokes velocity in the 3D VF method was over the crest of the sandbar, where the maximum breaking process occurred (Kumar et al., 2012). The disparity of the simulated vertical component of Stokes drifts originated from alternative assumptions in the paired models, which are presumed to be nil in the 3D RS concept and calculated as the divergence of horizontal Stokes mass flux in the 3D VF theory (i.e., $w^{S t}=-\nabla_{\perp} \cdot \int_{-h}^{z} \overrightarrow{u^{S t}} d z^{\prime}$ of Eq. (16)). It is not 


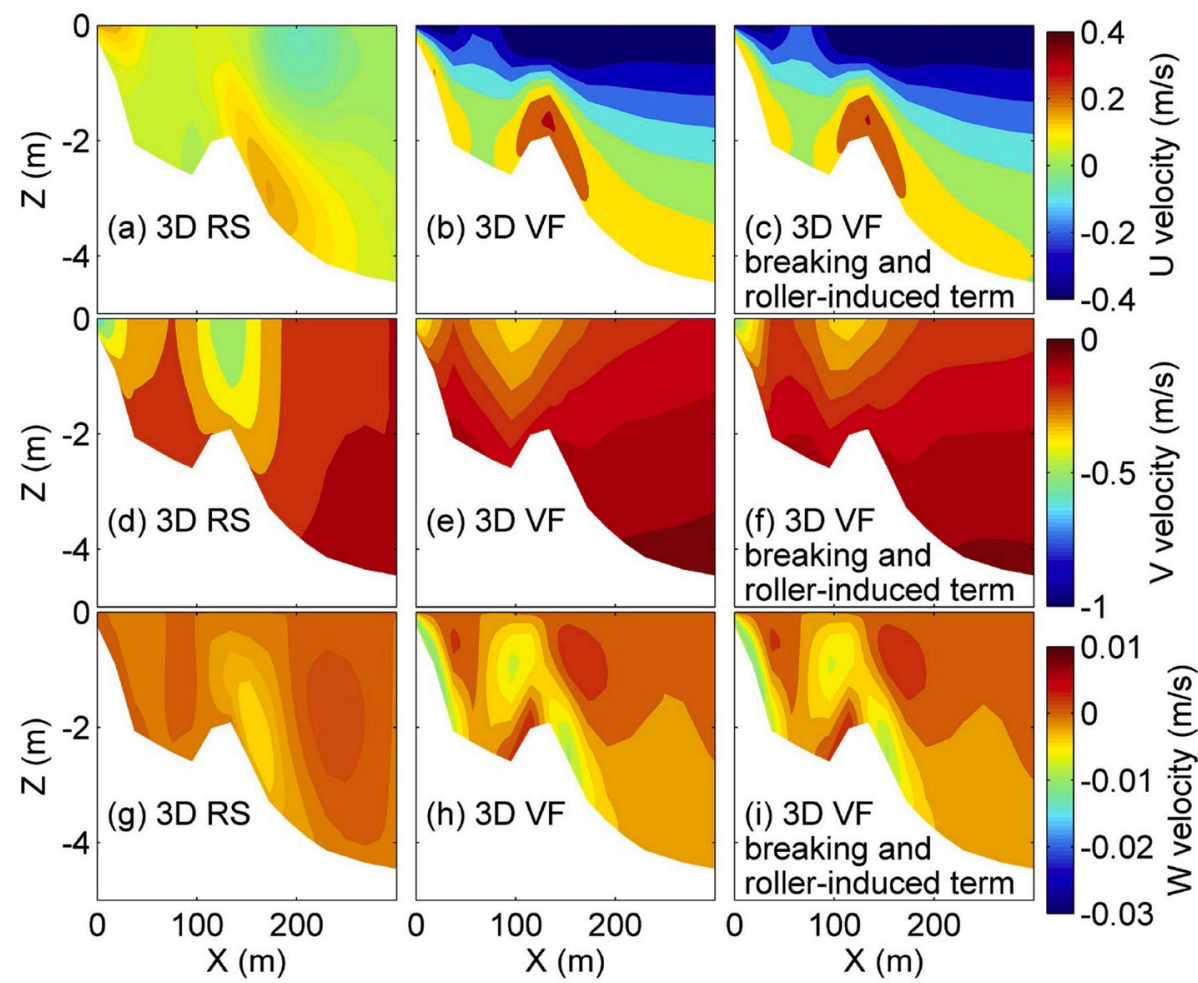

Fig. 10. Cross-shore sections of (a)-(c) cross-shore, (d)-(f) longshore, and (g)-(i) vertical Eulerian currents from the (a), (d) and (g) 3D RS, (b), (e), and (h) VF, and (c), (f) and (i) breaking and roller-induced accelerations in the 3D VF methods.

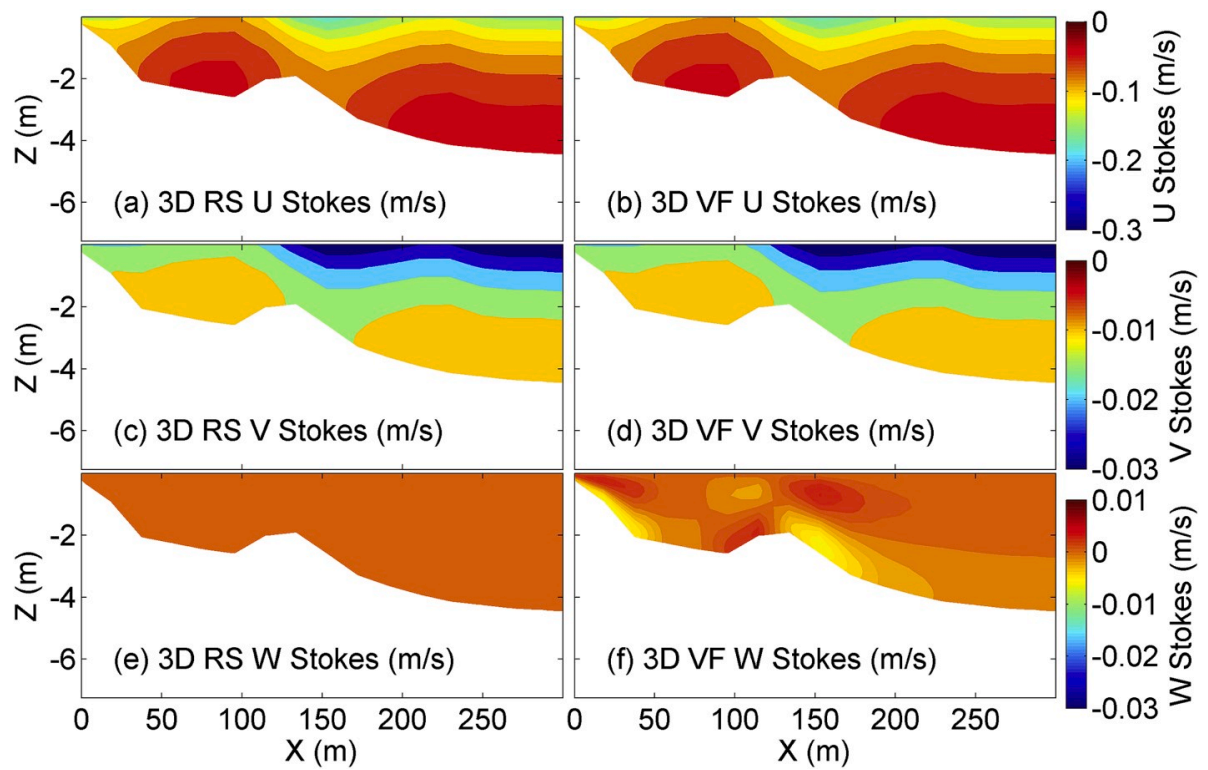

Fig. 11. Cross-shore sections of wave-induced (a) and (b) cross-shore, (c) and (d) longshore Stokes drifts, and (e) and (f) vertical Stokes drifts from the (a), (c), and (e) 3D RS and (b), (d), and (f) VF methods.

the purpose of this study to theoretically argue whether the vertical Stokes drift is nil or not (Mellor, 2003, 2017; McWilliams et al., 2004; Ardhuin et al., 2017), but that to compare the wave-induced circulation between both models directly. It can be concluded that both models have reproduced wave-induced effects successfully in the idealized inlet and planar and natural barred beaches. Further efforts on improving the 3D RS theory (Mellor, 2015; Ji et al., 2017) would be worthwhile to better capture the flow pattern of surface onshore and bottom undertow in the case of obliquely incident waves, but it is beyond the scope of this study.

\section{Summary and conclusions}

In this study, the recent 3D radiation stress (RS) theory and vortexforce (VF) formalism that account for wave-current interaction have been successfully implemented to the unstructured-grid FVCOM. Waveinduced circulation from the paired methods was compared in the idealized inlet, planar beach, and natural barred beach. The model was 
validated against observed wave and current data in the complex barred beach experiment. It should be noted that both methods have limitations, and for the detailed discussion of the underlying assumption and correctness, refer to Ardhuin et al. (2017) and Mellor (2015, 2017). The purpose of this study is to directly compare the wave-induced circulation using an unstructured-grid model, which is fundamental for its practical and realistic oceanographic applications in the future.

In the application of the wave-current coupled models to the idealized inlet, the 3D VF-induced depth-averaged circulation is found to be intensive in front of the inlet, while the 3D RS-generated flows are discernable near barriers and lateral boundaries. In the 3D VF approach, the breaking and roller-induced effects are primarily responsible for the wave-induced circulation. Simulations from both methods demonstrate that wave-current interaction near the surface layer is significantly stronger than that close to the bottom, and the horizontal Eulerian currents and wave-induced horizontal Stokes drifts follow the hyperbolic functions in the vertical space. In the case of the planar beach with obliquely incident waves along the open boundary, the paired models produce the surface onshore and bottom undertow currents successfully. However, this flow pattern in the 3D RS method extends further towards the offshore region as a result of unrealistic offshore currents generated by the vertical wave flux. Both models reproduce wave breaking and roller-induced longshore currents successfully, and their intensities simulated from the 3D VF model are stronger than those from the 3D RS model. Additionally, both modeling systems yield horizontal Stokes drifts reasonably, which is dominated by the cross-shore component and decreased monotonically from the surface to bottom layers.

Applications of the paired wave-current coupled models to the natural barred beach show that both methods capture wave dynamics and complex 3D circulation satisfactorily (e.g., surface onshore and bottom undertow currents). As a result of unphysical offshore currents from the shore in the 3D RS method, the location of this two-way flow pattern shifts further away from the shore to the sandbar's front. The wave breaking and roller-induced longshore current is well reproduced by two wave-current coupled models, and it is slightly stronger from the 3D RS method than that from the 3D VF method. Both approaches replicate the horizontal Stokes drifts comparably well, which are dominated by the onshore component and decrease monotonically from the surface to bottom. In all three applications, the 3D VF generates stronger vertical shear of the horizontal Eulerian velocity than that of the 3D RS, leading to the appreciable surface onshore and bottom undertow currents with obliquely incidence waves in the cases of planar and natural barred beaches.

Although the 3D RS method implemented in the modeling system showed some shortcomings, it can reproduce longshore currents and surface onshore and bottom undertow in the cross-shore direction successfully. Future work on comparing this modeling system with the modified 3D RS method (Ji et al., 2017) in FVCOM would be worthwhile. Compared to previous wave-current coupling to FVCOM (e.g., Ji et al., 2019), the novelty of this study is that both formalisms are successfully implemented in the two-way, dynamically coupled FVCOM/SWAVE system, and a direct comparison of the performance and physics effects between the RS and VF is conducted for various nearshore applications. The 3D VF simulations in this study are physically reasonable in a wide range of applications (Uchiyama et al., 2010; Kumar et al., 2012; Moghimi et al., 2013), which makes it a useful tool for prospective applications to complex systems in practical coastal ocean modeling. As the 3D unstructured-grid model is designed for nearshore and coastal seas with complex bathymetry and substantial wave effects on littoral processes, the implementations of the paired 3D wave-current interaction prescriptions into FVCOM can greatly merit the coastal ocean modeling community. It would be worthwhile to design additional numerical experiments including the case of non-breaking shoaling waves on gradually varying bathymetry (Ardhuin et al., 2008; Bennis et al., 2011; Kumar et al., 2011) and a more integrated system (Wang et al., 2017; Mao and Xia, 2018). With more observations of 3D current profiles in the surf zone, the practicability of the paired methodologies applied to the more complicated and realistic systems will be examined in the future.

\section{Declaration of Competing Interest}

The authors declare that they do not have any competing financial or associated interests that could have appeared to represent a conflict of interest in connection with this submitted paper.

\section{CRediT authorship contribution statement}

Meng Xia: Writing - original draft. Miaohua Mao: Writing - original draft. Qianru Niu: Writing - original draft.

\section{Acknowledgments}

This work is partially supported by the US NSF (No. 1856630). We would like to thank Drs. S. Moghimi of NOAA, Y. Uchiyama of Kobe Univ., S. Elgar of Woods Hole Oceanographic Institution, and F. Ardhuin of the Univ. of Brest, CNRS, IRD, Ifremer for constructive discussion of wave-current interaction. The DUCK94's data provided by Drs. Elgar (https://pv-lab.org/DUCK'94/) and Moghimi are greatly appreciated, and computations were supported by CISL's Cheyenne Supercomputer.

\section{Appendix A. Supplementary data}

Supplementary data to this article can be found online at https://doi. org/10.1016/j.ecss.2020.106771.

\section{References}

Aiki, H., Greatbatch, R.J., 2013. The vertical structure of the surface wave radiation stress for circulation over a sloping bottom as given by thickness-weighted-mean theory. J. Phys. Oceanogr. 43, 149-164.

Ardhuin, F., Jenkins, A.D., Belibassakis, K.A., 2008. Comments on "The three dimensional current and surface wave equations". J. Phys. Oceanogr. 38, 1340-1350.

Ardhuin, F., Suzuki, N., McWilliams, J.C., Aiki, H., 2017. Comments on "A combined derivation of the integrated and vertically resolved, coupled wave-current equations". J. Phys. Oceanogr. 47 (9), 2377-2385.

Benetazzo, A., Carniel, S., Sclavo, M., Bergamasco, A., 2013. Wave-current interaction: effect on the wave field in a semi-enclosed basin. Ocean Modell 70, 152-165.

Bennis, A.-C., Ardhuin, F., Dumas, F., 2011. On the coupling of wave and threedimensional circulation models: Choices of theoretical framework, practical implementation and adiabatic tests. Ocean Modell. 40, 260-272.

Bolaños, R., Brown, J.M., Souza, A.J., 2014. Wave-current interactions in a tide dominated estuary. Cont. Shelf Res. 87, 109-123.

Brown, J.M., Bolaños, R., Wolf, J., 2013. The depth-varying response of coastal circulation and water levels to $2 \mathrm{D}$ radiation stress when applied in a coupled wave-tide-surge modelling system during an extreme storm. Coast. Eng. 82, 102-113.

Chen, Q., Dalrymple, R.A., Kirby, J.T., Kennedy, A.B., Haller, M.C., 1999. Boussinesq modeling of a rip current system. J. Geophys Res. 104 (C9), 20,617-620,637.

Chen, C., Beardsley, Cowles, G., Qi, J., Lai, Z., Gao, G., Stuebe, D., Liu, H., Xu, Q., Xue, P., Ge, J., Ji, R., Hu, S., Tian, R., Huang, H., Wu, L., Lin, H., Sun, Y., Zhao, L., 2013. An unstructured-grid. In: Finite-Volume Community Ocean Model FVCOM User Manual, third ed. Univ. of Mass.-Dartmouth, New Bedford, Massachusetts, p. 404. SMAST/ UMASSD Tech. Rep.-13-0701.

Chen, J., Weisberg, R.H., Liu, Y., Zheng, L., Zhu, J., 2019. On the momentum balance of Tampa Bay. J. Geophys. Res. 124, 4492-4510.

Craik, A.D., Leibovich, S., 1976. A rational model for Langmuir circulation. J. Fluid Mech. 73 (3), 401-426.

Dodet, G., Bertin, X., Bruneau, N., Fortunato, A.B., Nahon, A., Roland, A., 2013. Wavecurrent interactions in a wave-dominated tidal inlet. J. Geophys. Res. 118 (3), 1587-1605.

Donelan, M.A., Dobson, F.W., Smith, S.D., Anderson, R.J., 1993. On the dependence of sea surface roughness on wave development. J. Phys. Oceanogr. 23 (9), 2143-2149.

Elgar, S., Guza, R.T., Raubenheimer, B., Herbers, T.H.C., Gallagher, E.L., 1997. Spectral evolution of shoaling and breaking waves on a barred beach. J. Geophys. Res. 102 (C7), 15797-15805.

Feddersen, F., Guza, R.T., Elgar, S., Herbers, T.H.C., 1998. Alongshore momentum balances in the nearshore. J. Geophys. Res. 103 (C8), 15667-15676.

Galperin, B., Kantha, L.H., Hassid, S., Rosati, A., 1988. A quasi-equilibrium turbulent energy model for geophysical flows. J. Atmos. Sci. 45 (1), 55-62. 
Haas, K.A., Warner, J.C., 2009. Comparing a quasi-3D to a full 3D nearshore circulation model: SHORECIRC and ROMS. Ocean Modell. 26 (1-2), 91-103.

Ji, C., Zhang, Q., Wu, Y., 2017. Derivation of three-dimensional radiation stress based on Lagrangian solutions of progressive waves. J. Phys. Oceanogr. 47 (11), 2829-2842.

Ji, C., Zhang, Q., Wu, Y., 2019. A comparison study of three-dimensional radiation stress formulations. Coast. Eng. J. 61 (2), 224-240.

Jiang, L., Xia, M., 2016. Dynamics of the Chesapeake Bay outflow plume: realistic plume simulation and its seasonal and interannual variability. J. Geophys. Res. 121, 1424-1445.

Kang, X., Xia, M., Pitula, J.S., Chigbu, P., 2017. Dynamics of water and salt exchange at Maryland Coastal Bays. Estuar. Coast. Shelf Sci. 189, 1-16.

Kennedy, A.B., Kirby, J.T., Gobbi, M.F., 2002. Simplified higher-order Boussinesq equations: I. Linear simplifications. Coast. Eng. 44 (3), 205-229.

Kumar, N., Voulgaris, G., Warner, J.C., 2011. Implementation and modification of a three-dimensional radiation stress formulation for the surf zone and rip-current applications. Coast. Eng. 58 (12), 1097-1117.

Kumar, N., Voulgaris, G., Warner, J.C., Olabarrieta, M., 2012. Implementation of the vortex force formalism in the coupled ocean-atmosphere-wave-sediment transport (COAWST) modeling system for inner shelf and surf zone applications. Ocean. Modell. 47, 65-95.

Lane, E.M., Restrepo, J.M., McWilliams, J.C., 2007. Wave-current interaction: a comparison of radiation-stress and vortex-force representations. J. Phys. Oceanogr. 37, 1122-1141.

Lin, P., Liu, P.L.F., 1998. A numerical study of breaking waves in the surf zone. J. Fluid Mech. 359, 239-264.

Liu, Y., MacCready, P., Hickey, B.M., Dever, E.P., Kosro, P.M., Banas, N.S., 2009. Evaluation of a coastal ocean circulation model for the Columbia River plume in summer 2004. J. Geophys. Res. 114, C00B04.

Liu, Y., Weisberg, R.H., Zheng, L., 2019. Impacts of hurricane Irma on the circulation and transport in Florida Bay and the Charlotte Harbor estuary. Estuar. Coast. https://doi. org/10.1007/s12237-019-00647-6.

Longuet-Higgins, M.S., 1970. Longshore currents generated by obliquely incident sea waves, 1 and 2. J. Geophys. Res. 75, 6778-6801.

Longuet-Higgins, M.S., Stewart, R.W., 1962. Radiation stress and mass transport in gravity waves, with application to 'surf beats'. J. Fluid. Mech. 13 (4), 481-504.

Longuet-Higgins, M.S., Stewart, R.W., 1964. Radiation stresses in water waves; a physical discussion, with applications. Deep Sea Res. 11, 529-562.

Madsen, O.S., 1994. Spectral wave-current bottom boundary layer flows. In: Coastal Engineering 1994. Proceedings of the 24th Internal Conference on Coastal Engineering Research Council, pp. 384-398. Kobe, Japan.

Mao, M., Xia, M., 2017. Dynamics of wave-current-surge interactions in Lake Michigan: a model comparison. Ocean. Modell. 110, 1-20.

Mao, M., Xia, M., 2018. Wave-current dynamics and interactions near the two inlets of the shallow lagoon-inlet-coastal ocean system under hurricane conditions. Ocean. Modell. 129, 124-144.

McWilliams, J.C., Restrepo, J.M., Lane, E.M., 2004. An asymptotic theory for the interaction of waves and currents in coastal waters. J. Fluid Mech. 511, 135-178.

Mellor, G.L., 2003. The three-dimensional current and surface wave equations. J. Phys. Oceanogr. 33, 1978-1989.

Mellor, G.L., 2005. Some consequences of the three-dimensional current and surface wave equations. J. Phys. Oceanogr. 35 (11), 2291-2298.

Mellor, G.L., 2008. The depth-dependent current and wave interaction equations: a revision. J. Phys. Oceanogr. 38, 2587-2596.

Mellor, G.L., 2013. Waves, circulation and vertical dependence. Ocean Dyn. 63 (4), 447-457.

Mellor, G.L., 2015. A combined derivation of the integrated and vertically-resolved, coupled wave-current equations. J. Phys. Oceanogr. 45, 1453-1463.

Mellor, G.L., 2016. On theories dealing with the interaction of surface waves and ocean circulation. J. Geophys. Res. 121, 4474-4486.
Mellor, G.L., 2017. Reply to "Comments on 'A combined derivation of the integrated and vertically resolved, coupled wave-current equations'”. J. Phys. Oceanogr. 47 (9), 2387-2389.

Michaud, H., Marsaleix, P., Leredde, Y., Estournel, C., Bourrin, F., Lyard, F., Mayet, C., Ardhuin, F., 2012. Three-dimensional modelling of wave-induced current from the surf zone to the inner shelf. Ocean Sci. 8, 657-681.

Moghimi, S., Klingbeil, K., Gräwe, U., Burchard, H., 2013. A direct comparison of a depth-dependent Radiation stress formulation and a Vortex force formulation with a three-dimensional coastal ocean model. Ocean Modell. 70, 132-144.

Newberger, P.A., Allen, J.S., 2007. Forcing a three-dimensional, hydrostatic, primitiveequation model for application in the surf zone: 2. Application to DUCK94. J. Geophys. Res. 112 (C8).

Nielsen, C., Apelt, C., 2003. The application of wave induced forces to a two-dimensional finite element long wave hydrodynamic mode. Ocean Eng. 30 (10), 1233-1251.

Niu, Q., Xia, M., 2016. Wave climatology of Lake Erie based on an unstructured-grid wave model. Ocean Dyn. 66 (10), 1271-1284.

Niu, Q., Xia, M., 2017. The role of wave-current interaction in Lake Erie's seasonal and episodic dynamics. J. Geophys. Res. 122, 7291-7311.

Niu, Q., Xia, M., Rutherford, E.S., Mason, D.M., Anderson, E.J., Schwab, D.J., 2015. Investigation of interbasin exchange and interannual variability in Lake Erie using an unstructured-grid hydrodynamic model. J. Geophys. Res. 120 (3), 2212-2232.

Olabarrieta, M., Warner, J.C., Kumar, N., 2011. Wave-current interaction in Willapa Bay. J. Geophys. Res. 116, C12014.

Phillips, O.M., 1977. The Dynamics of the Upper Ocean. Cambridge University Press, p. 336.

Qi, J., Chen, C., Beardsley, R.C., Perrie, W., Cowles, G.W., Lai, Z., 2009. An unstructuredgrid finite-volume surface wave model (FVCOM-SWAVE): implementation, validations and applications. Ocean Modell. 28 (1-3), 153-166.

Smagorinsky, J., 1963. General circulation experiments with the primitive equations: I. The basic experiment. Mon. Weather Rev. 91 (3), 99-164.

Svendsen, I.A., 1984. Wave heights and set-up in a surf zone. Coast. Eng. 8 (4), 303-329.

Svendsen, I.A., Haas, K., Zhao, Q., 2002. Quasi-3D Nearshore Circulation Model SHORECIRC, User's Manual. Center for Applied Coastal Research, Department of Civil Engineering, University of Delaware, Newark.

Ting, F.C.K., Kirby, J.T., 1994. Observations of undertow and turbulence in a laboratory surf zone. Coast. Eng. 24 (1-2), 51-80.

Tolman, H.L., 1990. The influence of unsteady, depths and currents of tides on windwave propagation in shelf seas. J. Phys. Oceanogr. 20, 1166-1174.

Uchiyama, Y., McWilliams, J.C., Shchepetkin, A.F., 2010. Wave-current interaction in an oceanic circulation model with a vortex-force formalism: application to the surf zone. Ocean Modell. 34 (1-2), 16-35.

Wang, J., Shen, Y., 2011. Development and validation of a three-dimensional, wavecurrent coupled model on unstructured meshes. Sci. China. Phys. Mech. Astron. 54 (1), 42-58.

Wang, P., Sheng, J., Hannah, C., 2017. Assessing the performance of formulations for nonlinear feedback of surface gravity waves on ocean currents over coastal waters. Cont. Shelf Res. 146, 102-117.

Wu, L., Chen, C., Guo, P., Shi, M., Qi, J., Ge, J., 2011. A FVCOM-based unstructured grid wave, current, sediment transport model, I. model description and validation. J. Ocean Univ. China 10, 1-8.

Zhang, Y., Chen, C., Beardsley, R.C., Perrie, W., Gao, G., Zhang, Y., Qi, J., Lin, H., 2020. Application of an unstructured grid surface wave model (FVCOM-SWAVE) to the Arctic Ocean: the interaction between ocean waves and sea ice. Ocean Modell. 145, 101532.

Zheng, P., Li, M., van der A, D.A., van der Zanden, J., Wolf, J., Chen, X., Wang, C., 2017. A 3D unstructured grid nearshore hydrodynamic model based on the vortex force formalism. Ocean Modell. 116, 48-69. 